\title{
Rotating Elastic Bodies in Einstein Gravity
}

\author{
LARS ANDERSSON \\ Albert Einstein Institute \\ ROBERT BEIG \\ Institut für Theoretische Physik der Universität Wien \\ AND \\ BERND G. SCHMIDT \\ Albert Einstein Institute
}

\begin{abstract}
We prove that, given a stress-free, axially symmetric elastic body, there exists, for sufficiently small values of the gravitational constant and of the angular frequency, a unique stationary, axisymmetric solution to the Einstein equations coupled to the equations of relativistic elasticity with the body performing rigid rotations around the symmetry axis at the given angular frequency. (C) 2009 Wiley Periodicals, Inc.
\end{abstract}

\section{Introduction}

In paper [1], we constructed for the first time static, self-gravitating elastic bodies in general relativity with no symmetries. Here we build on the ideas and techniques introduced in that paper to construct solutions to the Einstein equations describing steady states of self-gravitating matter in rigid rotation. The matter model we use is, as in [1], that of a perfectly elastic solid. We make the minimal symmetry assumptions necessary for a steady state in rigid rotation; namely, we assume that the reference body has an axis of symmetry. Further, we assume that the elastic material is isotropic. This condition, which was not needed in the static case, is necessary for our construction in the case of a rotating body.

The only class of solutions to the stationary Einstein equations with rotating matter previously known are the rotating perfect fluid solutions constructed by Heilig [9] for a certain class of equations of state. In the Newtonian theory, existence of steady states of self-gravitating perfect fluids in rigid rotation was established by Lichtenstein; see [8] for a modern presentation, and by Beig and Schmidt [5] for the case of elastic matter. All these solutions, including the ones constructed in the present paper, are, in addition to being stationary, also axisymmetric.

In Newtonian theory, two families of nonaxisymmetric rotating fluid configurations in explicit form are known; see [15] and references therein. These families 
of solutions are the Dedekind ellipsoids and the Jacobi ellipsoids, which in the language of general relativity have helical but no stationary or axial symmetry.

One expects asymptotically flat, rotating solutions of the Einstein equations, which are not axially symmetric, to be radiating and hence nonstationary. However, if one relaxes the condition that asymptotic flatness holds in the usual sense, it may be possible to construct helically symmetric solutions of the Einstein equations that are not axially symmetric. See [2] for a study of helically symmetric solutions in the special relativistic case. An argument to the effect that axisymmetry necessarily holds for a rotating fluid in general relativity was given by Lindblom [14], with the assumption that the fluid is viscous.

Equilibrium states of fluids or collisionless matter play an important role in astrophysics, providing the basic models of stars and galaxies. Depending on the equation of state-or in the case of collisionless matter, on the properties of the distribution function - a steady state may describe a compact body or a configuration where the matter density is nowhere vanishing. Typically, the objects of interest are compact.

In addition to fluids and collisionless matter, elastic bodies are of considerable interest in astrophysics in view of the fact that there are strong theoretical reasons for supposing that neutron stars have a solid crust, modeled by elastic matter; cf., e.g., $[6,7,10]$. The solutions that have been constructed in the just-mentioned papers are all spherically symmetric, although perturbation analyses have been carried out, allowing for axial perturbations to break the spherical symmetry [11].

\subsection{Rotating Bodies in Elasticity}

Elastic matter is, as discussed in Section 2.1 below, described by a map $f^{A}$ from spacetime to a three-dimensional manifold, called material manifold or body, whose points label the particles making up the elastic continuum, and which is taken to be a connected, bounded domain in flat $\mathbb{R}^{3}$.

In considering a rotating steady state, it is important to distinguish between the microscopic and macroscopic degrees of freedom. The microscopic degrees of freedom of the elastic matter are described by the configuration $f^{A}$, while the macroscopic aspects are described by the stress energy tensor generated by the matter and the metric of the spacetime containing the body. For a rotating body in equilibrium, it is the case that the stress energy tensor, as well as the spacetime metric, are stationary, i.e., invariant under the flow of a Killing vector $\xi^{\mu} \partial_{\mu}=\partial_{t}$, called the stationary Killing vector, while the matter particles, described by the configuration map, are in motion relative to $\partial_{t}$.

As mentioned above, the rotating bodies we construct are axially symmetric. In particular, the spacetime containing the body admits a Killing field $\eta^{\mu} \partial_{\mu}=\partial_{\phi}$, called the axial Killing vector, which commutes with $\xi^{\mu}$. In addition, there is a constant $\Omega$, the angular frequency of rotation, such that the matter particles move along the helical orbits of the Killing vector $\xi^{\mu}+\Omega \eta^{\mu}$; i.e., the configuration $f^{A}$ is constant along the flow of the helical field. 
It is nevertheless the case, assuming axisymmetry of the configuration and that the elastic material is isotropic and frame indifferent (cf. Section 2.1) that all spacetime tensors naturally derived from the configuration are both axisymmetric and stationary. This applies, for example, to the matter flow vector induced by the configuration, the stress tensor, or, in fact, to the full stress energy tensor.

We now briefly describe the method used in this paper. Consider a Cauchy surface $M$ transverse to the stationary Killing field. The equations for the gravitational field variables are derived by imposing the condition of stationarity and restricting the Einstein equations, reduced in harmonic gauge, to $M$. No axisymmetry condition is imposed at this stage.

The Einstein equations imply, via the Bianchi identity, a set of equations for the matter variables. These equations are derived by considering a configuration that is comoving with respect to the helical flow and restricting it to $M$. Here the axial vector field $\eta^{\mu}$ is assumed to be specified in advance. There are, a priori, four matter equations for the three unknowns $f^{A}$. We deal with this problem by simply dropping one of the four equations. It turns out, however, that this supplementary equation follows from the others when $\eta^{\mu}$ is Killing, as is the case for a solution to the system derived by the above procedure.

The resulting coupled system of equations, assuming standard constitutive conditions for the elastic material, is elliptic for sufficiently small values of $\Omega$. The system depends on the parameters $G$ and $\Omega$. We look for solutions to this system for small, nonzero values of $G$ and $\Omega$, near the background solution given by taking the spacetime to be Minkowski, the configuration to be stress-free, and the Newton constant $G$ and $\Omega$ to be both 0 .

The boundary between the matter region and the vacuum region depends on the unknown configuration. To deal with this problem, we write the equations in material form in a way analogous to [1] and apply the implicit function theorem. This cannot be done directly due to the failure of the linearized operator to be invertible. This is a standard problem for elasticity with natural boundary conditions, i.e., vanishing normal stress at the boundary. Following [1], what we actually solve is a projected version of the field equations such that the implicit function theorem does apply. One must then show, as in fact turns out to be the case, that the solution to this projected system is actually a solution to the full system.

So far the vector field $\eta^{\mu}$ has been essentially arbitrary except for the condition that it commute with $\xi^{\mu}$. In order to ensure that $\eta^{\mu}$ be a Killing vector, we now assume that the material manifold is axisymmetric as a subset of Euclidean $\mathbb{R}^{3}$ and that $\eta^{\mu}$ is the pullback of the axial vector field on the body under the trivial configuration. It then follows by uniqueness that the vector field $\eta^{\mu}$ is a Killing vector.

It now remains to show that the solution found by the implicit function argument satisfies the Einstein equations. In particular, the gauge conditions must be satisfied and the elastic equation must be valid in its original form. This condition is equivalent to the condition that a certain linear system of equations coming 
from the Bianchi identities has only the trivial solution. In fact, the linear system under discussion is homogenous precisely because the Killing nature of $\eta^{\mu}$ guarantees that the above-mentioned supplementary equation is satisfied, provided that the main elastic equation is valid. The rest of the argument essentially follows the pattern of [1], where this is referred to as the equilibration argument, and we shall use this terminology here as well.

\subsection{Outline of the Paper}

In Section 2.1, we give some background on relativistic elasticity. Section 2.3 introduces stationary metrics and defines the field variables $h_{i k}, U$, and $\psi_{i}$ used to parametrize the spacetime metric. In contrast to the static case, there is a further component $\psi_{i}$ of the gravitational field, corresponding to the failure of $\xi^{\mu}$ to be hypersurface orthogonal in general. Next, in Section 2.4, the stationary Einstein equations are written in terms of the field variables just introduced; this corresponds effectively to performing a Kaluza-Klein reduction.

The field equations imply a set of integrability conditions, which are derived in Section 2.5. One of these identities is the elasticity equation, which is later used as one of the set of equations to be solved using the implicit function theorem. The rotation of the elastic body is introduced in Section 2.6. This is done by choosing a spacelike vector field $\eta^{\mu} \partial_{\mu}$ that commutes with the stationary Killing vector $\xi^{\mu}$ and assuming that $f^{A}, \mu\left(\xi^{\mu}+\Omega \eta^{\mu}\right)$ is 0 . It will later turn out that $\eta^{\mu}$ is actually the axial Killing vector.

In Section 2.7, the stress tensor is expressed in terms of the geometric variables and the configuration $f^{A}$. In particular, this allows us to write the components of the stress energy tensor in terms of the field variables and obtain (2.37), the basic PDE system for $h_{i k}, U, \psi_{i}, f^{A}$. Because some of the equations are not elliptic we use, as in the static case, harmonic coordinates to extract an elliptic system. In the stationary case, it is necessary to make explicit use also of the condition that the time function be harmonic; cf. Section 2.8. Finally, we have reduced the field equations to an elliptic free boundary value problem in space. In order to avoid dealing directly with the free boundary aspect of the problem, we move all equations to the body. This is done in Section 2.9, following closely the procedure in [1]. The final details needed to completely specify the PDE problem to be solved are introduced in Section 2.10. There we introduce the relaxed state and a flat metric on the body. We assume that the shape of the body is axisymmetric and use the relaxed configuration to define the vector field $\eta^{i}$ in space. We also introduce the assumption that the elastic material is isotropic.

As in the static case considered in [1], we must consider a projected system in order to be able to apply the implicit function theorem. The analytical aspects of this problem are considered in Section 3. The solution to the projected system is then shown in Section 4 to be a solution to the full set of field equations in the body frame and to be axisymmetric. In Section 4.1 we move the projected equations 
and solutions back to space. The vector field $\eta^{i}$ is proved to be a Killing field in Section 4.2.

In Section 4.3, we derive some divergence identities that play an essential role in the equilibration argument. This leads up to the main theorem, Theorem 4.6, which is stated and proved in Section 4.4. In particular, we prove that the harmonic coordinate conditions are satisfied for the solution of the reduced system that has been constructed, and hence that we have solved the full set of field equations.

The spacetimes constructed in Theorem 4.6 have an isometry group $\mathbb{R} \times \mathbb{S}^{1}$ generated by the commuting Killing fields $\xi^{\mu}$ and $\eta^{\mu}$. For spacetimes with two commuting Killing fields, it was first proved by Papapetrou [17] in vacuum and by Kundt and Trümper [13] for fluids that orthogonal transitivity holds. Recall that orthogonal transitivity is the condition that the distribution of 2-surface elements perpendicular to the generators of the symmetry group is surface-forming. This condition is used for constructing Weyl-type coordinates, which play a dominant role in attempts in the exact solution literature to find rotating body solutions; see [18] and references therein.

In Section 4.5, we establish that for the spacetimes constructed in Theorem 4.6, the distribution defined by the 2-surface elements orthogonal to the group orbit for the action of the stationary and axisymmetric Killing vector is integrable. In the case of a smooth spacetime, where the Frobenius theorem applies directly, this fact implies that orthogonal transitivity holds, i.e., that there are 2 -surfaces perpendicular to the generators of the symmetry group. In the present case, however, this step needs further analysis, which we defer to a later paper.

\section{Field Equations of a Rotating, Self-Gravitating Elastic Body}

\subsection{Relativistic Elasticity}

Let $\left(\mathcal{M}, g_{\mu \nu}\right)$ be a $(3+1)$-dimensional spacetime. The body $\mathcal{B}$ is a 3-manifold, possibly with boundary. We shall consider the case when $\mathcal{B}$ is a bounded domain in the extended body $\mathbb{R}_{\mathcal{B}}^{3}$. The body domain $\mathcal{B}$ is assumed to have a smooth boundary. In this paper, we shall only consider the case where $\mathcal{B}$ is connected. The fields considered in elasticity are configurations $f: \mathcal{M} \rightarrow \mathcal{B}$ and deformations $\phi: \mathcal{B} \rightarrow$ $\mathcal{M}$, with the property that $f \circ \phi=\mathbf{i d}_{\mathcal{B}}$.

Let $t$ be a time function on $M$ and introduce a $(3+1)$ split $\mathcal{M}=\mathbb{R} \times M$. We consider coordinates $\left(x^{\mu}\right)=\left(t, x^{i}\right)$ on $\mathcal{M}$, where $x^{i}$ are coordinates on the space manifold $M$. On $\mathbb{R}_{\mathcal{B}}^{3}$ we use coordinates $X^{A}$. The body $\mathcal{B}$ is endowed with metric $\delta_{\mathcal{B}}$ and a compatible volume form $V_{A B C}$. We assume that in a suitable Cartesian coordinate system $\delta_{\mathcal{B}}$ has components $\delta_{A B}$ where $\delta_{A B}$ is the Kronecker delta and $V_{123}=1$.

The configuration $f: \mathcal{M} \rightarrow \mathcal{B}$ is by assumption a submersion. The derivative of $f$ is assumed to have a timelike kernel; i.e., there is a unit timelike vector field $u^{\mu}$ on $f^{-1}(\mathcal{B})$ with $u_{\mu} u^{\mu}=-1$ such that

$$
u^{\mu} f_{, \mu}^{A}=0 \text {. }
$$


The field $u^{\mu}$ is the velocity field of the matter and describes the trajectories of the material particles.

Let $\Lambda=\Lambda(f, \partial f, g)$ be the energy density for the elastic material in its own rest frame. The Lagrange density for the self-gravitating elastic body now takes the form

$$
\mathcal{L}=-\frac{R_{g} \sqrt{-g}}{16 \pi G}+\Lambda \sqrt{-g}
$$

The Einstein equations resulting from the variation of the action with respect to $g^{\mu v}$ take the form

$$
G_{\mu \nu}=8 \pi G T_{\mu \nu},
$$

where $G_{\mu \nu}$ is the Einstein tensor of $g_{\mu \nu}$ and $T_{\mu \nu}$ is the stress energy tensor of the material, given by

$$
T_{\mu \nu}=2 \frac{\partial \Lambda}{\partial g^{\mu \nu}}-\Lambda g_{\mu \nu}
$$

On the other hand, the canonical stress energy tensor is given by

$$
\mathcal{T}_{\mu}{ }^{\nu}=\frac{\partial \Lambda}{\partial f^{A}, \nu} f_{, \mu}^{A}-\delta_{\mu}{ }^{\nu} \Lambda
$$

General covariance implies, by the Rosenfeld-Belinfante theorem, that

$$
\mathcal{T}_{\mu \nu}=-T_{\mu \nu}
$$

see [12, sec. 7].

Given a configuration $f^{A}\left(x^{\mu}\right)$, define $\gamma^{A B}=f^{A}{ }_{, \mu} f^{B}{ }_{, \nu} g^{\mu \nu}$, and let $\gamma_{A B}$ be the inverse of $\gamma^{A B}$. General covariance implies that $\Lambda$ is of the form $\Lambda=$ $\Lambda\left(f^{A}, \gamma^{A B}\right)$; cf. [12, sec. 7]; see also [4, sec. 4]. A stored energy function of this form is said to satisfy material frame indifference. If in addition, as we shall later assume, $\Lambda$ depends only on the principal invariants $\lambda_{i}, i=1,2,3$, of $\gamma^{A B}$, defined as the elementary symmetric polynomials in the eigenvalues of $\gamma^{A}{ }_{B}=$ $\gamma^{A C}\left(\delta_{\mathcal{B}}\right)_{C B}$, then the material is called isotropic.

Define

Then we have

$$
S_{A B}=2 \frac{\partial \Lambda}{\partial \gamma^{A B}}-\Lambda \gamma_{A B}
$$

$$
T_{\mu \nu}=\Lambda u_{\mu} u_{\nu}+S_{\mu \nu},
$$

where $S_{\mu \nu}=S_{A B} f^{A}{ }_{, \mu} f^{B}{ }_{, \nu}$. In particular, $S_{\mu \nu} u^{\nu}=0$. The relativistic number density $n_{g}$ is defined by

$$
n_{g}^{2}=\frac{1}{3 !} V_{A B C} V_{A^{\prime} B^{\prime} C^{\prime}} \gamma^{A A^{\prime}} \gamma^{B B^{\prime}} \gamma^{C C^{\prime}} .
$$

We have $n_{g}=\left(\operatorname{det} \gamma^{A B}\right)^{1 / 2}$ and hence

$$
\frac{\partial n_{g}}{\partial \gamma^{A B}}=\frac{1}{2} n_{g} \gamma_{A B} .
$$


Define the stored energy function $\epsilon$ by

$$
\Lambda=n_{g} \epsilon,
$$

and the elastic stress tensor $\tau_{A B}$ by

$$
\tau_{A B}=2 \frac{\partial \epsilon}{\partial \gamma^{A B}} .
$$

With these definitions, $S_{A B}$ takes the form

$$
S_{A B}=n_{g} \tau_{A B}
$$

and we can write

$$
T_{\mu \nu}=n_{g} \in u_{\mu} u_{\nu}+n_{g} \tau_{A B} f_{, \mu}^{A} f_{, \nu}^{B} .
$$

See [19] for a more explicit expression of $T_{\mu \nu}$ in terms of the invariants $\left(\lambda_{i}\right)$.

If material frame indifference holds, then if $\Lambda$ is viewed as a functional of $f^{A}, g_{\mu \nu}$, we have that for any spacetime diffeomorphism $\sigma$,

$$
\Lambda\left[f \circ \sigma, \sigma^{*} g\right]=\Lambda[f, g] \circ \sigma
$$

and hence all spacetime quantities constructed from $\Lambda, f^{A}$, and $g_{\mu \nu}$ are covariant under $\sigma$, including $n_{g}, u^{\mu}$, and $\tau_{A B} f^{A}{ }_{, \mu} f^{B}{ }_{, \nu}$. In particular, this also holds for $T_{\mu \nu}$.

Let $\Sigma$ be an isometry of $\left(\mathcal{B}, \delta_{\mathcal{B}}\right)$. The matrix $\left(\Sigma_{*} \gamma\right)^{A}{ }_{B}$ is related to $\gamma^{A}{ }_{B}$ by an orthogonal similarity transform and hence has the same invariants $\lambda_{i}$ as $\gamma^{A}{ }_{B}$. Hence, for an isotropic material,

$$
\Lambda[\Sigma \circ f, g]=\Lambda[f, g] .
$$

\subsection{Material and Spacetime Isometries}

We now introduce the notion of symmetry of a configuration that will play an important role in this paper. Suppose the spacetime $(\mathcal{M}, g)$ has an isometry $\sigma$. Then $\sigma$ defines a material symmetry of $f^{A}$ if there is an isometry $\Sigma$ of $\left(\mathcal{B}, \delta_{\mathcal{B}}\right)$ such that

$$
\Sigma \circ f=f \circ \sigma
$$

Thus, in particular, if the configuration is comoving with an isometry, i.e., if $u^{\mu}$ is proportional to a Killing vector $\xi^{\mu}$, then the configuration has the flow $\sigma_{s}$ of $\xi^{\mu}$ as a material isometry with $\Sigma$ given by the identity map on $\mathcal{B}$, in which case it follows that $f^{A} \circ \sigma_{s}=f^{A}$. However, this does not hold for a general one-parameter family of material isometries. It follows from the last two statements in the previous subsection that, for an isotropic material, a spacetime isometry $\sigma$ that also defines a material isometry leaves the Lagrangian invariant, i.e., $\Lambda[f, g]=\Lambda[f, g] \circ \sigma$, and thus $T_{\mu \nu}$ is also invariant under $\sigma$, i.e., $\sigma^{*} T=T$.

The following is an example that is relevant to our situation. Suppose we have two timelike Killing vectors $\xi^{\mu}$ and $\xi^{\prime \mu}$. In the situation considered here, the interesting case is where $\xi^{\mu}$ is the stationary Killing field, while $\xi^{\prime \mu}=\xi^{\mu}+\Omega \eta^{\mu}$ is the helical Killing field. Then one may consider the case where the configuration 
is comoving with respect to $\xi^{\prime \mu}$ while the flow $\sigma_{s}$ of $\xi^{\mu}$ defines a configuration symmetry in the sense that there is a one-parameter family of isometries $\Sigma_{S}$ of the body such that

$$
\Sigma_{s} \circ f^{A}=f^{A} \circ \sigma_{s}
$$

In this case, $\Sigma_{s}$ are rotations of the body. We see from the above that it is possible for the configuration to explicitly depend on the Killing time $t$, defined with respect to $\xi^{\mu}$, although $T_{\mu \nu}$ is independent of $t$.

\subsection{Stationary Metrics}

We now assume that $(\mathcal{M}, g)$ is stationary; i.e., there is a timelike Killing field $\xi^{\mu} \partial_{\mu}=\partial_{t}$. Further, we assume the space manifold $M$ to be diffeomorphic to $\mathbb{R}^{3}$. It will sometimes be convenient to denote this space by $\mathbb{R}_{\mathcal{S}}^{3}$. Define a function $U=\frac{1}{2} \ln \xi^{\mu} \xi_{\mu}$ and a 1-form $\psi=\psi_{i} d x^{i}$ such that $e^{-2 U} \xi_{\mu} d x^{\mu}=d t+\psi$. Then $g$ can be written in the form

$$
g_{\mu \nu} d x^{\mu} d x^{\nu}=-e^{2 U}\left(d t+\psi_{i} d x^{i}\right)^{2}+e^{-2 U} h_{i j} d x^{i} d x^{j},
$$

where $h_{i j} d x^{i} d x^{j}$ is a metric on the level sets of $t$, and $U, \psi_{i}$, and $h_{i j}$ are time independent. The inverse metric takes the form

$$
g^{\mu \nu} \partial_{\mu} \partial_{\nu}=-e^{-2 U} \partial_{t}^{2}+e^{2 U} h^{i j}\left(\partial_{i}-\psi_{i} \partial_{t}\right)\left(\partial_{j}-\psi_{j} \partial_{t}\right),
$$

where $h^{i j} h_{j k}=\delta^{i}{ }_{k}$. The spacetime volume element is given by

$$
\sqrt{-g}=e^{-2 U} \sqrt{h}
$$

Let $\square_{g}$ be the d'Alembertian in $(\mathcal{M}, g)$. The assumption that $\xi^{\mu} \partial_{\mu}=\partial_{t}$ is a Killing vector implies

$$
\square_{g} t=-e^{2 U} D^{i} \psi_{i}, \quad \square_{g} x^{i}=-e^{2 U} h^{j k} \Gamma_{j k}^{i},
$$

where $D_{i}$ and the Christoffel symbols refer to $h_{i j}$.

\subsection{Kaluza-Klein Reduction} by

$$
R_{g} \sqrt{-g}=\sqrt{h}\left(R_{h}+2 \Delta_{h} U-2|D U|_{h}^{2}+e^{4 U}|\omega|_{h}^{2}\right) .
$$

Here $|D U|_{h}^{2}=D_{k} U D^{k} U$ and similarly for $|\omega|_{h}^{2}$. Define $H^{A B}$ by

$$
\gamma^{A B}=e^{2 U} H^{A B} .
$$

The reduced number density $n$ is defined with respect to $H^{A B}$,

$$
n^{2}=\frac{1}{3 !} V_{A B C} V_{A^{\prime} B^{\prime} C^{\prime}} H^{A A^{\prime}} H^{B B^{\prime}} H^{C C^{\prime}} .
$$


Then we have

$$
\begin{aligned}
\frac{\partial n}{\partial H^{A B}} & =\frac{1}{2} n H_{A B}, \\
n_{g} & =e^{3 U} n,
\end{aligned}
$$

so that with the form (2.4) for $g$, we have

$$
\Lambda \sqrt{-g}=n \epsilon e^{U} \sqrt{h} .
$$

Taking into account the fact that the term $2 \Delta_{h} U$ in the scalar curvature expression (2.8) contributes a total divergence to the action and can be dropped, we may now write the action in the reduced form

$$
\mathcal{L}=-\frac{\sqrt{h}}{16 \pi G}\left(R_{h}-2|D U|_{h}^{2}+e^{4 U}|\omega|_{h}^{2}\right)+\rho e^{U} \sqrt{h},
$$

where $\rho=n \epsilon$.

Let $G_{i j}=R_{i j}-\frac{1}{2} R h_{i j}$ be the Einstein tensor of $h_{i j}$ and define

$$
\Theta_{i j}=\frac{1}{4 \pi G}\left[\left(D_{i} U\right)\left(D_{j} U\right)-\frac{1}{2} h_{i j}\left(D_{k} U\right)\left(D^{k} U\right)\right]
$$

and

$$
\Omega_{i j}=\frac{1}{4 \pi G} e^{4 U}\left[\frac{1}{4} h_{i j} \omega_{k l} \omega^{k l}-\omega_{i k} \omega_{j}^{k}\right] .
$$

The reduced field equations now take the form

$$
\begin{gathered}
\Delta_{h} U=4 \pi G e^{U}\left(\rho+\frac{\partial \rho}{\partial U}\right) \chi_{f^{-1}(\mathcal{B})}-e^{4 U} \omega_{k l} \omega^{k l}, \\
D^{i}\left(e^{4 U} \omega_{i j}\right)=-8 \pi G e^{U} \frac{\partial \rho}{\partial \psi^{j}} \chi_{f^{-1}(\mathcal{B})} \\
G_{i j}=8 \pi G\left(\Theta_{i j}+\Omega_{i j}+e^{U}\left(2 \frac{\partial \rho}{\partial h^{i j}}-\rho h_{i j}\right) \chi_{f^{-1}(\mathcal{B})}\right) .
\end{gathered}
$$

In (2.14) we have used the indicator function $\chi_{f^{-1}(\mathcal{B})}$ of the body to make explicit the support of $\rho$. Define $\tau, \tau_{i}$, and $\tau_{i j}$ by

$$
T_{\mu \nu}=\tau\left(d t+\psi_{i} d x^{i}\right)^{2}+2 \tau_{j} d x^{j}\left(d t+\psi_{i} d x^{i}\right)+\tau_{i j} d x^{i} d x^{j} .
$$

Then we have the following:

LEMMA 2.1

$$
\begin{aligned}
e^{U}\left(2 \frac{\partial \rho}{\partial h^{i j}}-\rho h_{i j}\right) & =\tau_{i j}, \\
e^{U} \frac{\partial \rho}{\partial \psi^{i}} & =-\tau_{i}, \\
e^{U}\left(\rho+\frac{\partial \rho}{\partial U}\right) & =e^{-4 U} \tau+\tau_{\ell}^{\ell} .
\end{aligned}
$$


For the proof of Lemma 2.1, see the appendix. After substituting (2.16) into (2.14), the reduced field equations take the form ${ }^{1}$

$$
\begin{aligned}
\Delta_{h} U & =4 \pi G \chi_{f^{-1}(\mathcal{B})}\left(e^{-4 U} \tau+\tau_{k}{ }^{k}\right)-e^{4 U} \omega_{k l} \omega^{k l}, \\
D^{i}\left(e^{4 U} \omega_{i j}\right) & =8 \pi G \chi_{f^{-1}(\mathcal{B})} \tau_{j}, \\
G_{i j} & =8 \pi G\left(\chi_{f^{-1}(\mathcal{B})} \tau_{i j}+\Theta_{i j}+\Omega_{i j}\right) .
\end{aligned}
$$

\subsection{Integrability Conditions}

The quantities $\Theta_{i j}$ and $\Omega_{i j}$ satisfy the identities

$$
8 \pi G D^{j} \Theta_{i j}=2\left(D_{i} U\right) \Delta_{h} U,
$$

and, by using $D_{[i} \omega_{j k]}=0$,

$$
8 \pi G D^{j} \Omega_{i j}=2\left[e^{4 U}\left(D_{i} U\right) \omega_{k l} \omega^{k l}-\omega_{i k} D^{j}\left(e^{4 U} \omega_{j}^{k}\right)\right] .
$$

In case $G \neq 0$, we obtain from (2.17b) the integrability conditions

$$
D^{i} \tau_{i}=0,
$$

together with the boundary condition

$$
\left.\tau_{i} n^{i}\right|_{f^{-1}(\partial \mathcal{B})}=0
$$

Further, we have

$$
D^{j} \tau_{i j}-2 \omega_{i j} \tau^{j}=-\left(D_{i} U\right)\left(e^{-4 U} \tau+\tau_{k}^{k}\right)
$$

and

$$
\left.\tau_{i j} n^{j}\right|_{f^{-1}(\partial \mathcal{B})}=0
$$

as a consequence of the contracted Bianchi identities for $h_{i j}$ applied to the lefthand side of (2.17c), together with (2.18), (2.19), and (2.17a).

\subsection{Implementing Rotation}

Define a vector field $\eta^{\mu}$ by

$$
\eta^{\mu} \partial_{\mu}=\eta^{i} \partial_{i}
$$

The scalar product $\alpha=g_{\mu \nu} \xi^{\mu} \eta^{\nu}$ satisfies

$$
e^{-2 U} \alpha+\psi_{j} \eta^{j}=0
$$

Since by assumption $(\mathcal{M}, g)$ is stationary with respect to $\xi^{\mu}$, it holds that $\eta^{\mu}$ commutes with $\xi^{\mu}$ if and only if $\eta^{i}$ does not depend on $t$. In particular, in the case

\footnotetext{
${ }^{1}$ Equation (2.17b) corrects a typo in [3, (2.47)].
} 
we are considering, the vector field $\eta^{\mu}$ is itself a Killing vector if and only if the equations

$$
\begin{aligned}
\mathcal{L}_{\eta} U & =0, \\
\mathcal{L}_{\eta} \psi_{i} & =0, \\
\mathcal{L}_{\eta} h_{i j} & =0,
\end{aligned}
$$

hold. In these expressions the operator $\mathcal{L}_{\eta}$ means the Lie derivative of the respective object with respect to $\eta^{k} \partial_{k}$. Note that (2.26b) implies

$$
2 \omega_{i j} \eta^{j}+D_{i}\left(e^{-2 U} \alpha\right)=0 .
$$

Define the velocity field $u^{\mu}$ by

$$
u^{\mu}=b^{-1}\left(\xi^{\mu}+\Omega \eta^{\mu}\right),
$$

where $\Omega$ is a real parameter corresponding to the rotation speed, and $b$ is a normalizing factor, determined by $u^{\mu} u_{\mu}=-1$ in $f^{-1}(\mathcal{B})$. It is important to note here that the rotational field $\xi^{\mu}+\Omega \eta^{\mu}$ in general will fail to be globally timelike for nonzero values of $\Omega$. However, for a suitable range of $\Omega$, it makes sense to require $\xi^{\mu}+\Omega \eta^{\mu}$ to be timelike in the body.

We now impose rotation of the body by requiring that the configuration $f^{A}$ satisfy the condition $u^{\mu} f^{A}, \mu=0$, i.e.,

$$
f_{, \mu}^{A}\left(\xi^{\mu}+\Omega \eta^{\mu}\right)=0 .
$$

Since $T_{\mu \nu} u^{\nu}=-\rho u_{\mu}$, due to (2.1) the stress energy tensor satisfies the relation

$$
u_{[\mu} T_{\nu] \rho} u^{\rho}=0 .
$$

It follows from (2.30) that

$$
\left(u_{0} T_{i \mu}-u_{i} T_{0 \mu}\right) u^{\mu}=0
$$

holds, which, by using (2.29), doing some cancellations, and multiplying by $e^{-2 U}$ gives

$$
\begin{aligned}
\left(1-\Omega e^{-2 U} \alpha\right)^{2} \tau_{i}+\Omega(1 & \left.-\Omega e^{-2 U} \alpha\right) \tau_{i j} \eta^{j} \\
& +\Omega e^{-4 U} \eta_{i}\left[\left(1-\Omega e^{-2 U} \alpha\right) \tau+\Omega \tau_{j} \eta^{j}\right]=0 .
\end{aligned}
$$

Equation (2.32) can be proved by explicit computation, by using (2.16) and (2.34). As a consequence of (2.32) we have the following:

LEMMA 2.2 For sufficiently small $\Omega$,

$$
\begin{aligned}
\left(1-\Omega e^{-2 U} \alpha\right) \tau_{i}+\Omega \tau_{i j} \eta^{j} & \\
& +\Omega e^{-4 U} \eta_{i}\left[\tau+\Omega\left(1-\Omega e^{-2 U} \alpha\right)^{-1} \tau_{j} \eta^{j}\right]=0 .
\end{aligned}
$$




\subsection{Stress Tensor}

In order to write the field equations, we shall need the stress tensor for the elastic material. For consistency with the treatment of the static case considered in [1], we shall here make use of an analogous form of the stress tensor. Recall that assuming material frame indifference, the stored energy function $\epsilon$ is a function of $f^{A}$ and $\gamma^{A B}=f^{A}{ }_{, \mu} f^{B}{ }_{, \nu} g^{\mu \nu}$. Taking equation (2.29) into account, we find

$$
\begin{aligned}
\gamma^{A B}=f_{, i}^{(A}{ }^{B)}{ }_{, j}\left[e^{2 U} h^{i j}+2 \Omega e^{2 U} \psi^{i} \eta^{j}\right. \\
\left.+\Omega^{2}\left(e^{2 U} \psi^{k} \psi_{k}-e^{-2 U}\right) \eta^{i} \eta^{j}\right] .
\end{aligned}
$$

In the computations below we make use of $H^{A B}$ defined by $\gamma^{A B}=e^{2 U} H^{A B}$, so that

$$
H^{A B}=f_{, i}^{(A} f^{B)}{ }_{j}\left[h^{i j}+2 \Omega \psi^{i} \eta^{j}+\Omega^{2}\left(\psi^{k} \psi_{k}-e^{-4 U}\right) \eta^{i} \eta^{j}\right] .
$$

Let

$$
\begin{gathered}
\sigma_{A B}=-2 \frac{\partial \epsilon}{\partial H^{A B}}, \quad \sigma_{\mu \nu}=n f_{, \mu}^{A} f_{, \nu}^{B} \sigma_{A B}, \\
\sigma_{\mu}{ }^{A}=f^{B}{ }_{, \mu} \sigma_{B C} H^{C A} .
\end{gathered}
$$

It follows from the definition that

$$
\sigma_{A B}=-2 e^{-2 U} \frac{\partial \epsilon}{\partial \gamma^{A B}} .
$$

Our next task is to evaluate the dependence on $\Omega$ of the terms occurring in the left-hand side of (2.16). It is straightforward to verify that the following lemma holds:

LEMMA 2.3 There are $z, z_{i}$, and $z_{i j}$ depending smoothly on $f^{A}, g_{\mu \nu}$, and their first derivatives, as well as $\Omega$ and $G$, such that the following equations are valid:

$$
\begin{aligned}
e^{U}\left(2 \frac{\partial \rho}{\partial h^{i j}}-\rho h_{i j}\right) & =-e^{U}\left(\sigma_{i j}-\Omega z_{i j}\right), \\
e^{U} \frac{\partial \rho}{\partial \psi^{i}} & =-e^{U} \Omega z_{i}, \\
e^{U}\left(\rho+\frac{\partial \rho}{\partial U}\right) & =e^{U}\left(n \epsilon-\sigma_{\ell}^{\ell}+\Omega z\right) .
\end{aligned}
$$

By the results of Lemma 2.3 and Lemma 2.1, we have

$$
\tau_{i j}=-e^{U}\left(\sigma_{i j}-\Omega z_{i j}\right) .
$$

We are now able to rewrite the integrability condition (2.22) in the form

$$
D^{j}\left(e^{U} \sigma_{i j}\right)=e^{U}\left(n \epsilon-\sigma_{\ell}^{\ell}\right) D_{i} U+\Omega\left[D^{j}\left(e^{U} z_{i j}\right)+2 e^{U} \omega_{i j} z^{j}+z D_{i} U\right] .
$$


Taking the above facts into account, we arrive at the system of equations

$$
\begin{aligned}
\Delta_{h} U= & \left.4 \pi G \chi_{f^{-1}(\mathcal{B})} e^{U}\left(n \epsilon-\sigma_{\ell}^{\ell}\right)+\Omega z\right)-e^{4 U} \omega_{k l} \omega^{k l}, \\
D^{i}\left(e^{4 U} \omega_{i j}\right)=8 \pi G \chi_{f}-1(\mathcal{B}) & e^{U} \Omega z_{j}, \\
G_{i j}= & 8 \pi G\left[-\chi_{f}^{-1}(\mathcal{B}) e^{U}\left(\sigma_{i j}-\Omega z_{i j}\right)+\Theta_{i j}+\Omega_{i j}\right], \\
D^{j}\left(e^{U} \sigma_{i j}\right)= & e^{U}\left(n \epsilon-\sigma_{\ell}^{\ell}\right) D_{i} U \\
& +\Omega\left[D^{j}\left(e^{U} z_{i j}\right)+2 e^{U} \omega_{i j} z^{j}+z D_{i} U\right] \text { in } f^{-1}(\mathcal{B}),
\end{aligned}
$$

subject to the boundary condition

$$
\left.\left(\sigma_{i j}-\Omega z_{i j}\right) n^{j}\right|_{\partial f^{-1}(\mathcal{B})}=0 .
$$

\subsection{Gauge Reduction}

Two of the equations in system (2.37) fail to be elliptic in the form given above, namely $(2.37 b)$ and $(2.37 c)$. The reason for this failure is related to the diffeomorphism invariance of the four-dimensional Einstein equations. As in the static case, the method that shall be used to avoid this problem is to make use of harmonic coordinates.

Taking into account the fact that $g_{\mu \nu}$ is stationary, we have

$$
\begin{aligned}
\square_{g} t & =\frac{1}{\sqrt{-g}} \partial_{\mu}\left(g^{\mu \nu} \sqrt{-g} \partial_{\nu}\right) t \\
& =e^{2 U} D^{i} \psi_{i} .
\end{aligned}
$$

Thus, $e^{2 U} D^{i} \psi_{i}=0$ precisely when the time $t$ is harmonic.

The left-hand side of equation (2.37b) is of the form

$$
D^{i}\left(e^{4 U} \omega_{i j}\right)=e^{4 U}\left[4 D^{i} U \omega_{i j}+\frac{1}{2}\left(\Delta \psi_{j}-R_{j}{ }^{k} \psi_{k}\right)\right]-\frac{1}{2} e^{4 U} D_{j} D^{i} \psi_{i}
$$

The term $D_{j} D^{i} \psi_{i}$ causes this expression to fail to be an elliptic in $\psi_{i}$. However, the following reduced form of equation (2.37b),

$$
D^{i}\left(e^{4 U} \omega_{i j}\right)+\frac{1}{2} D_{j}\left(e^{4 U} D_{i} \psi^{i}\right)=8 \pi G \chi_{f^{-1}(\mathcal{B})} e^{U} \Omega z_{j},
$$

which modifies the left-hand side by a quantity that vanishes if the harmonic time condition is satisfied, is elliptic in $\psi_{i}$.

Similarly, (2.37c) fails to be elliptic due to the covariance of $R_{i j}$. Following [1, sec. 3.1], let $V^{i}=h^{j k}\left(\Gamma_{j k}^{i}-\widehat{\Gamma}_{j k}^{i}\right)$ where $\hat{\Gamma}_{j k}^{i}$ are the Christoffel symbols of a fixed Euclidean background metric on $M$. Then $V^{i}=0$ is the condition for harmonic coordinates in $M$. By replacing $R_{i j}$ by $R_{i j}-D_{(i} V_{j)}$ we arrive, after rewriting equation $(2.37 \mathrm{c})$ by making use of the identity [1, (3.11)], at the reduced Einstein 
equation

$$
\begin{aligned}
& -\frac{1}{2} \Delta_{h} h_{i j}+Q_{i j}(h, \partial h) \\
& =-8 \pi G e^{U}\left(\sigma_{i j}-h_{i j} \sigma_{l}^{l}+\Omega\left(z_{i j}-h_{i j} z_{l}^{l}\right)\right) \chi_{f^{-1}(\mathcal{B})} \\
& \quad+2 D_{i} U D_{j} U+e^{4 U}\left[h_{i j} \omega_{k l} \omega^{k l}-2 \omega_{i k} \omega_{j}^{k}\right] .
\end{aligned}
$$

As in [1], we shall first solve the reduced system involving (2.40) and (2.39), and once the solution is in hand show that the solution to the reduced system is actually a solution to the full system. We construct solutions by an implicit function theorem argument applied to a projected version of the field equations in material form.

\subsection{Field Equations in Material Form}

In the Eulerian picture, the domain $f^{-1}(\mathcal{B})$ depends on the unknown configuration $f$. This introduces a "free boundary" aspect in the Eulerian version of the field equations, which we will avoid by passing to the material or Lagrangian form of the field equations. In this form of the equations, the configuration $f$ is replaced by the deformation $\phi$, and the entire system of field equations is moved to the extended body $\mathbb{R}_{\mathcal{B}}^{3}$. In particular, in this formulation, the elastic field equation lives on the fixed domain $\mathcal{B}$.

The Piola transform of $\sigma_{i}{ }^{j}$ is

$$
\bar{\sigma}_{i}{ }^{A}=J\left(f^{A}{ }_{, j} \sigma_{i}{ }^{j}\right) \circ \phi
$$

Similarly, we introduce the Piola transform of $z_{i j}$. Since $\mathcal{B}$ has a smooth boundary, there is a linear extension operator that takes functions on $\mathcal{B}$ to functions on $\mathbb{R}_{\mathcal{B}}^{3}$. In particular, this allows us to define an extension $\hat{\phi}$ of $\phi$ that is equal to $\mathbf{i}$ outside a compact set. We use $\hat{\phi}$ to move the fields from space to $\mathbb{R}_{\mathcal{B}}^{3}$ and use the bar notation introduced in [1, sec. 3.2] to denote the quantities transported under $\hat{\phi}$. In particular, we define

$$
\bar{U}=U \circ \hat{\phi}, \quad \overline{\partial_{i} U}=\partial_{i} U \circ \hat{\phi}, \quad \overline{\psi_{i}}=\psi_{i} \circ \hat{\phi}, \quad \overline{h_{i j}}=h_{i j} \circ \hat{\phi} .
$$

Note that for the barred quantities, it is the frame components that are pulled back and not the tensor itself. Equation (2.37a) in the material frame becomes

$$
\left.\overline{\Delta_{h} U}=4 \pi G \chi_{\mathcal{B}} e^{\bar{U}}\left(n \bar{\epsilon}-\bar{\sigma}_{\ell}{ }^{\ell}\right)+\Omega \bar{z}\right)-e^{4 \bar{U}} \overline{\omega_{k l} \omega^{k l}} \text { in } \mathbb{R}_{\mathcal{B}}^{3} .
$$

We remark that covariance of the Laplacian gives

$$
\overline{\Delta_{h} U}=\Delta_{\widehat{\phi}^{*} h}(U \circ \widehat{\phi}) .
$$

Next, equation (2.39) in the material frame becomes

$$
\overline{D^{i}\left(e^{4 U} \omega_{i j}\right)}+\frac{1}{2} \overline{D_{j}\left(e^{4 U} D_{i} \psi^{i}\right)}=8 \pi G \chi_{\mathcal{B}} e^{\bar{U}} \Omega \bar{z}_{j}
$$


Equation (2.40) becomes

$$
\begin{aligned}
-\frac{1}{2} & \overline{\Delta_{h} h_{i j}}+Q_{i j}(\bar{h}, \partial \bar{h}) \\
= & -8 \pi G e^{\bar{U}}\left(\bar{\sigma}_{i j}-\bar{h}_{i j} \bar{\sigma}_{l}{ }^{l}+\Omega\left(\bar{z}_{i j}-\bar{h}_{i j} \bar{z}_{l}^{l}\right)\right) \chi_{\mathcal{B}} \\
& +2 \overline{D_{i} U D_{j} U}+e^{4 \bar{U}}\left[\bar{h}_{i j} \overline{\omega_{k l} \omega^{k l}}-2-\overline{\omega_{i k} \omega_{j}{ }^{k}}\right] .
\end{aligned}
$$

Equations (2.37d) and (2.37e) become in the material frame

$$
\begin{aligned}
D_{A}\left(e^{\bar{U}} \sigma_{i}{ }^{A}\right)= & e^{\bar{U}}\left(\epsilon-\frac{\bar{\sigma}_{\ell}^{\ell}}{\bar{n}}\right) \overline{\partial_{i} U} \\
& +\Omega\left[D_{A}\left(e^{\bar{U}} \bar{z}_{i}{ }^{A}\right)+2 e^{\bar{U}} \overline{\frac{\omega_{i j} z^{j}}{\bar{n}}}+\bar{z} \overline{\partial_{i} U}\right] \text { in } \mathcal{B},
\end{aligned}
$$

subject to the boundary condition

$$
\left.\left(\bar{\sigma}_{i}^{A}-\Omega \bar{z}_{i}{ }^{A}\right) n_{A}\right|_{\partial \mathcal{B}}=0 .
$$

\subsection{Constitutive Conditions}

Similarly to the static case, we shall assume the existence of a relaxed reference configuration for the elastic material, which is such that suitable ellipticity properties hold for the elasticity operator evaluated in the relaxed state. The relaxed state is given by the body $\mathcal{B}$, a compact, connected domain $\mathcal{B} \subset \mathbb{R}_{\mathcal{B}}^{3}$ with smooth boundary $\partial \mathcal{B}$, together with a reference configuration $\mathbf{i}: \mathbb{R}_{\mathcal{B}}^{3} \rightarrow \mathbb{R}_{\mathcal{S}}^{3}$. We assume a reference Euclidean metric $\hat{\delta}$ on $M=\mathbb{R}_{\mathcal{S}}^{3}$ is given. The body metric $\mathbb{R}_{\mathcal{B}}^{3}$ on $\mathbb{R}_{\mathcal{B}}^{3}$ is defined by $\delta_{\mathcal{B}}=\mathbf{i}^{*} \hat{\delta}$. The relaxed nature of the reference configuration is expressed by the condition

$$
\left.\left(\frac{\partial \epsilon}{\partial H^{A B}}\right)\right|_{\left(U=0, H=\delta_{\mathcal{B}}\right)}=0 \text { in } \mathcal{B} .
$$

The specific rest mass, i.e., the rest mass term in the relativistic stored energy function, should obey

$$
\stackrel{\circ}{\epsilon}(X)=\left.\epsilon\right|_{\left(U=0, H=\delta_{\mathcal{B}}\right)} \geq C
$$

for some constant $C>0$. Further, we assume that the elastic material is such that there is a constant $C^{\prime}>0$ such that the pointwise stability condition

$$
\stackrel{\circ}{L}_{A B C D} N^{A B} N^{C D} \geq C^{\prime}\left(\delta_{C A} \delta_{B D}+\delta_{C B} \delta_{A D}\right) N^{A B} N^{C D} \text { in } \mathcal{B}
$$

holds, where

$$
\stackrel{\circ}{L}_{A B C D}(X):=\left.\left(\frac{\partial^{2} \epsilon}{\partial H^{A B} \partial H^{C D}}\right)\right|_{\left(U=0, H=\delta_{\mathcal{B}}\right)} .
$$

In the isotropic case considered in this paper, $\epsilon$ depends only on the invariants of $\gamma^{A B}=e^{2 U} H^{A B}$, defined with respect to the body metric $\left(\delta_{\mathcal{B}}\right)_{A B}$ (cf. Section 2.1). 
It follows that $\stackrel{\circ}{\epsilon}$ is independent of $X$ and there are constants $\stackrel{\circ}{\lambda}$ and $\stackrel{\circ}{v}$ so that

$$
\stackrel{\circ}{L}_{A B C D}=\stackrel{\circ}{\lambda} \delta_{A B} \delta_{C D}+2 \stackrel{\circ}{\mu} \delta_{C(A} \delta_{B) D},
$$

in terms of which condition (2.45) holds exactly when

$$
\stackrel{\circ}{\mu}>0, \quad 3 \stackrel{\circ}{\lambda}+2 \stackrel{\circ}{\mu}>0
$$

cf. [16, sec. 4.3]. The constants $\stackrel{\circ}{\lambda}$ and $\stackrel{\circ}{\mu}$ are apart from a common constant factor the classical Lamé moduli. Inequalities (2.48) are usually expressed by saying that the Poisson ratio defined by

$$
v=\frac{\stackrel{\circ}{\lambda}}{2(\stackrel{\circ}{\lambda}+\stackrel{\circ}{\mu})}
$$

satisfy $-1<v<\frac{1}{2}$. In fact, for most materials occurring in practice there holds $\frac{1}{4}<v<\frac{1}{3}$.

We shall assume that the body is axisymmetric. To make this notion concrete, let $x^{i}$ and $X^{A}$ be coordinates on $\mathbb{R}_{\mathcal{S}}^{3}$ and $\mathbb{R}_{\mathcal{B}}^{3}$, respectively, so that the Euclidean metrics $\hat{\delta}$ and $\delta_{\mathcal{B}}$ have components $\delta_{i j}$ and $\delta_{A B}$, respectively. The body $\mathcal{B}$ is axially symmetric if there is a one-parameter subgroup of Euclidean motions, defined with respect to $\delta_{A B}$, that leaves $\mathcal{B}$ invariant. We may without loss of generality assume that the subgroup leaving $\mathcal{B}$ invariant is generated by the Killing field

$$
\eta^{A} \partial_{A}=X^{2} \partial_{1}-X^{1} \partial_{2},
$$

which necessarily is such that $\eta^{A}$ is tangent to $\partial \mathcal{B}$. Given the axial Killing field $\eta^{A}$ on $\mathbb{R}_{\mathcal{B}}^{3}$, define a vector field $\eta^{i}$ on $\mathbb{R}_{\mathcal{S}}^{3}$ by

$$
\eta^{i} \partial_{i}=\mathbf{i}_{*}\left(\eta^{A} \partial_{A}\right)
$$

In particular, we may without loss of generality assume $\eta^{i} \partial_{i}$ to be of the form $\eta^{i} \partial_{i}=x^{2} \partial_{1}-x^{1} \partial_{2}$.

In addition to the above-mentioned conditions, we shall in the following assume that the elastic material is isotropic; cf. Section 2.1. Recall that if the elastic material is isotropic, then $\Lambda$ and hence also the stored energy function $\epsilon$ depends only on the invariants $\lambda_{i}$ of $\gamma^{A B}$, defined with respect to the body metric $\delta_{\mathcal{B}}$; cf. Section 2.1. Consequently, in view of the discussion above (see in particular Section 2.7), the reduced energy density $\rho=n \epsilon$ can be viewed as a function $\rho=\rho\left(\lambda_{i}\right)$.

The invariants $\lambda_{i}$ are functions of the form $\lambda_{i}=\lambda_{i}\left(f, \partial f, U, \psi_{i}, h_{i j} ; \eta^{i}, \Omega\right)$. In the present case, we are using a coordinate system on $\mathcal{B}$ in which the metric $\delta_{\mathcal{B}}$ has constant components and hence the $\lambda_{i}$ do not depend on $f$ but only on its derivatives. We may write $\rho$ as a functional $\rho=\rho[f, g ; \eta, \Omega]$, where the symbol $g$ is used as shorthand for the gravitational variables $U, \psi_{i}$, and $h_{i j}$ parametrizing the spacetime metric $g_{\mu \nu}$. 


\section{Analytical Setting}

We now introduce the analytical setting that will be used to construct solutions to the field equations. Fix a weight $\delta \in\left(-1,-\frac{1}{2}\right)$. Further, fix $p>3$. The parameters $\delta$ and $p$ will be used to determine the weighted Sobolev spaces that are used in the implicit function argument.

The system of equations in material form has the unknowns $\phi^{i}, U, \psi^{i}$, and $h_{i j}$. Let

$$
B_{1}=W^{2, p}(\mathcal{B}) \times W_{\delta}^{2, p} \times W_{\delta}^{2, p} \times E_{\delta}^{2, p},
$$

where $E_{\delta}^{2, p}$ is the space of asymptotically Euclidean metrics introduced in [1, sec. 2.3], and let

$$
B_{2}=\left[L^{p}(\mathcal{B}) \times B^{1-1 / p, p}(\partial \mathcal{B})\right] \times L_{\delta-2}^{p} \times L_{\delta-2}^{p} \times L_{\delta-2}^{p} .
$$

Thus, $B_{1}$ is a Banach manifold and $B_{2}$ is a Banach space.

The residuals of equations (2.44a) with boundary condition $(2.44 \mathrm{~b}),(2.41)$, (2.42), and (2.43), which depend on the Newton constant $G$ and the rotation velocity $\Omega$ as parameters define a map $\mathcal{F}: \mathbb{R}^{2} \times B_{1} \rightarrow B_{2}$. Thus, $\mathcal{F}$ has components $\left(\mathcal{F}_{\phi}, \mathcal{F}_{U}, \mathcal{F}_{\psi}, \mathcal{F}_{h}\right)$ corresponding to the components of $B_{2}$, given by

$$
\mathcal{F}_{\phi}=\left[\mathcal{F}_{\phi}^{\mathcal{B}}, \mathcal{F}_{\phi}^{\partial \mathcal{B}}\right]
$$

where

$$
\begin{aligned}
& \mathcal{F}_{\phi}^{\mathcal{B}}=D_{A}\left(e^{\bar{U}} \sigma_{i}{ }^{A}\right)-e^{\bar{U}}\left(\epsilon-\frac{\bar{\sigma}_{\ell}^{\ell}}{\bar{n}}\right) \overline{\partial_{i} U},
\end{aligned}
$$

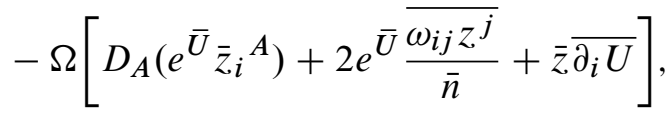

$$
\begin{aligned}
& \mathcal{F}_{\phi}^{\partial \mathcal{B}}=\left.\left(\bar{\sigma}_{i}{ }^{A}-\Omega \bar{z}_{i}{ }^{A}\right) n_{A}\right|_{\partial \mathcal{B}},
\end{aligned}
$$

and

$$
\begin{aligned}
\mathcal{F}_{U}= & \left.\overline{\Delta_{h} U}-4 \pi G \chi_{\mathcal{B}} e^{\bar{U}}\left(n \bar{\epsilon}-\bar{\sigma}_{\ell}^{\ell}\right)+\Omega \bar{z}\right)+e^{4 \bar{U}} \overline{\omega_{k l} \omega^{k l}}, \\
\mathcal{F}_{\psi}= & \overline{D^{i}\left(e^{4 U} \omega_{i j}\right)}+\frac{1}{2} \overline{D_{j}\left(e^{4 U} D_{i} \psi^{i}\right)}-8 \pi G \chi_{\mathcal{B}} e^{\bar{U}} \Omega \bar{z}_{j}, \\
\mathcal{F}_{h}= & -\frac{1}{2} \overline{\Delta_{h} h_{i j}}+Q_{i j}(\bar{h}, \partial \bar{h}) \\
& +8 \pi G e^{\bar{U}}\left(\bar{\sigma}_{i j}-\bar{h}_{i j} \bar{\sigma}_{l}^{l}+\Omega\left(\bar{z}_{i j}-\bar{h}_{i j} \bar{z}_{l}^{l}\right)\right) \chi_{\mathcal{B}} \\
& -2 \overline{D_{i} U D_{j} U}-e^{4 \bar{U}}\left[\bar{h}_{i j} \overline{\omega_{k l} \omega^{k l}}-2 \overline{\omega_{i k} \omega_{j}^{k}}\right] .
\end{aligned}
$$

We now have $\mathcal{F}=\mathcal{F}((G, \Omega),(\phi, \bar{U}, \bar{\psi}, \bar{h}))$. Write a general element of $B_{1}$ as $Z$. We will use the implicit function theorem to construct solutions to $\mathcal{F}=0$ for $G$ and $\Omega$ close to $0 \in \mathbb{R}^{2}$.

An essential assumption which allows us to introduce a relaxed configuration is that there is a reference Euclidean metric $\hat{\delta}$ on $M=\mathbb{R}_{\mathcal{S}}^{3}$, and a diffeomorphism 
$\mathbf{i}: \mathbb{R}_{\mathcal{B}}^{3} \rightarrow \mathbb{R}_{\mathcal{S}}^{3}$. As discussed in Section 2.10, an Euclidean metric on $\mathbb{R}_{\mathcal{B}}^{3}$ is defined by $\delta_{\mathcal{B}}=\mathbf{i}^{*} \hat{\delta}$. Recall that $\mathcal{B}$ is assumed to be a connected domain with smooth boundary.

From the constitutive conditions (cf. Section 2.10), we have that

$$
Z_{0}=\left(\mathbf{i}, 0,0, \hat{\delta}_{i j} \circ \mathbf{i}\right)
$$

is a solution to the equation $\mathcal{F}\left(0, Z_{0}\right)=0$. In order to apply the implicit function theorem at $\left(0, Z_{0}\right)$, it is necessary that the Frechet derivative $D_{2} \mathcal{F}\left(0, Z_{0}\right)$ be an isomorphism. We see that $\mathcal{F}(0, Z)$ is of the form

$$
\begin{aligned}
\mathcal{F}_{\phi}(0, Z)= & {\left[D_{A}\left(e^{\bar{U}} \sigma_{i}{ }^{A}\right)-e^{\bar{U}}\left(\epsilon-\frac{\bar{\sigma}_{\ell}^{\ell}}{\bar{n}}\right) \overline{\partial_{i} U},\left.\bar{\sigma}_{i}{ }^{A} n_{A}\right|_{\partial \mathcal{B}}\right], } \\
\mathcal{F}_{U}(0, Z)= & \overline{\Delta_{h} U}+e^{4 \bar{U}} \overline{\omega_{k l} \omega^{k l}}, \\
\mathcal{F}_{\psi}(0, Z)= & \overline{D^{i}\left(e^{4 U} \omega_{i j}\right)}+\frac{1}{2} \overline{D_{j}\left(e^{4 U} D_{i} \psi^{i}\right)}, \\
\mathcal{F}_{h}(0, Z)= & -\frac{1}{2} \overline{\Delta_{h} h_{i j}}+Q_{i j}(\bar{h}, \partial \bar{h})-2 \overline{D_{i} U D_{j} U} \\
& -e^{4 \bar{U}}\left[\bar{h}_{i j} \overline{\omega_{k l} \omega^{k l}}-2 \overline{\omega_{i k} \omega_{j}^{k}}\right] .
\end{aligned}
$$

It follows from the constitutive conditions stated in Section 2.10 that $D_{\phi} \mathcal{F}_{\phi}(0, Z)$ is elliptic.

\subsection{Projected System}

An analysis along the lines of [1, sec. 4.2] shows that $D_{2} \mathcal{F}\left(0, Z_{0}\right)$ is of the form

$$
\left(\begin{array}{cccc}
D_{\phi} \mathcal{F}_{\phi} & D_{U} \mathcal{F}_{\phi} & 0 & D_{h} \mathcal{F}_{\phi} \\
0 & \Delta & 0 & 0 \\
0 & 0 & \frac{1}{2} \Delta & 0 \\
0 & 0 & 0 & -\frac{1}{2} \Delta
\end{array}\right),
$$

where the entries are evaluated at $Z_{0}$. The diagonal entries are isomorphisms between the weighted spaces given in the definitions of $B_{1}$ and $B_{2}$, with the exception of $D_{\phi} \mathcal{F}_{\phi}$. As in the static case this has a nontrivial kernel and cokernel; see the discussion in [1, sec. 4]. The kernel and cokernel can be identified with the space of Killing fields on $\left(\mathcal{B}, \delta_{\mathcal{B}}\right)$. Therefore, in order to construct solutions, we will consider the projected system

$$
\mathbb{P}_{\mathcal{B}} \mathcal{F}=0,
$$

where $\mathbb{P}_{\mathcal{B}}: B_{2} \rightarrow B_{2}$ is a projection operator that is defined exactly along the lines of [1, sec. 4]. In particular, $\mathbb{P}_{\mathcal{B}}$ is defined to act as the identity in the second to fourth components of $B_{2}$, while in the first component of $B_{2}$ it acts as the unique projection along the cokernel of $D_{\phi} \mathcal{F}_{\phi}\left(0, Z_{0}\right)$ onto the range of $D_{\phi} \mathcal{F}_{\phi}\left(0, Z_{0}\right)$, which leaves the boundary data in the first component of $B_{2}$ unchanged. We use the label $\mathcal{B}$ to indicate that $\mathbb{P}_{\mathcal{B}}$ operates on fields on the body and the extended body. We shall later need to transport the projection operator to fields on $\mathbb{R}_{\mathcal{S}}^{3}$. 
Let $\left(b_{i}, \tau_{i}\right)$ denote pairs of elements in $W^{2, p}(\mathcal{B}) \times W^{1-1 / p, p}(\partial \mathcal{B})$. The restriction of $\mathbb{P}_{\mathcal{B}}$ to the first component of $B_{2}$, which we here denote by the same symbol, is defined by setting $\mathbb{P}_{\mathcal{B}}\left(b_{i}, \tau_{i}\right)=\left(b_{i}^{\prime}, \tau_{i}\right)$, satisfying

$$
\int_{\mathcal{B}} \xi^{i} b^{\prime}{ }_{i}=\int_{\partial \mathcal{B}} \xi^{i} \tau_{i}
$$

for all Killing fields $\xi^{i}$. Pairs $\left(b^{\prime}{ }_{i}, \tau_{i}\right)$ satisfying this condition are called equilibrated. As discussed in [1, sec. 4], the definition of $\mathbb{P}_{\mathcal{B}}$ implies there is a unique $\eta_{i}$ of the form $\eta_{i}=\alpha_{i}+\beta_{i j} X^{j}$ for constants $\alpha_{i}$ and $\beta_{i j}$ satisfying $\beta_{i j}=-\beta_{j i}$ such that

$$
b^{\prime}{ }_{i}=b_{i}-\eta_{i} \chi_{\mathcal{B}}
$$

We further restrict the domain of $\mathbb{P}_{\mathcal{B}} \mathcal{F}$ to eliminate the kernel of $D_{\phi} \mathbb{P}_{\mathcal{B}} \mathcal{F}$. By assumption (cf. Section 2.10), $\mathcal{B}$ has an axis of symmetry, which without loss of generality can be identified with the $X^{3}$-axis. Fix a point $X_{0}$ on the axis of symmetry of $\mathcal{B}$; i.e., $X_{0}$ has coordinates $\left(0,0, X^{3}\right)$ for some $X^{3}$. Recall that the kernel of $D_{\phi} \mathcal{F}$ consists of the Killing fields of $\left(\mathcal{B}, \delta_{\mathcal{B}}\right)$. A killing field in $\mathcal{B}$ is determined by specifying its value and antisymmetrized derivative at one point. Following the proof of [1, prop. 4.3], ${ }^{2}$ define $\mathbb{X}$ to be the submanifold of $B_{1}$ such that

$$
(\phi-\mathbf{i})\left(X_{0}\right)=0 \quad \text { and } \quad \delta_{i}^{C} \delta_{C[A} \partial_{B]}(\phi-\mathbf{i})^{i}\left(X_{0}\right)=0 .
$$

and define $\mathbb{Y}$ to be the range of the projection operator $\mathbb{P}_{\mathcal{B}}$. An application of the implicit function theorem to the map

$$
\mathbb{P}_{\mathcal{B}} \mathcal{F}: \mathbb{X} \rightarrow \mathbb{Y}
$$

now gives the following result, analogous to [1, prop. 4.3].

Proposition 3.1 Let $\mathcal{F}: B_{1} \rightarrow B_{2}$ be the map defined by (3.1) and let $\mathbb{P}_{\mathcal{B}}$ be defined as in [1, sec. 4.3]. Then for sufficiently small values of Newton's constant $G$ and the rotation velocity $\Omega$, there is a unique solution $Z=Z(G, \Omega)$, where $Z=$ $\left(\phi, \bar{U}, \bar{\psi}_{i}, \overline{h_{i j}}\right)$, to the reduced, projected equation for a self-gravitating rotating elastic body given by

$$
\mathbb{P}_{\mathcal{B}} \mathcal{F}((G, \Omega), Z)=0,
$$

which satisfies condition (3.3). In particular, for any $\epsilon>0$, there are $G>0$ and $\Omega>0$ such that $Z(G, \Omega)$ satisfies the inequality

$$
\|\phi-\mathbf{i}\|_{W^{2, p}(\mathcal{B})}+\left\|\overline{h_{i j}}-\delta_{i j}\right\|_{W_{\delta}^{2, p}}+\|\bar{U}\|_{W_{\delta}^{2, p}}+\|\bar{\psi}\|_{W_{\delta}^{2, p}}<\epsilon .
$$

The proof of Proposition 3.1 proceeds along exactly the same lines as the proof of [1, prop. 4.3] and is left to the reader.

\footnotetext{
${ }^{2}$ The discussion here corrects some typos in the proof of [1, prop. 4.3]; in particular, the antisymmetrization in (3.3) corrects the corresponding expression in [1].
} 


\section{Equilibration}

Arguing along the lines of [1, sec. 5], we have the following corollary to Proposition 3.1:

COROLlaRY 4.1 For any $\epsilon>0$, there are $G>0$ and $\Omega>0$ such that the inequality

$$
\|\phi-\mathbf{i}\|_{W^{2, p}(\mathcal{B})}+\left\|h_{i j}-\delta_{i j}\right\|_{W_{\delta}^{2, p}}+\|U\|_{W_{\delta}^{2, p}}+\|\psi\|_{W_{\delta}^{2, p}}<\epsilon
$$

holds.

\subsection{Eulerian Form of the Projected Equations}

Let $\mathbb{P}_{f^{-1}(\mathcal{B})}$ be the Eulerian form of the projection operator, defined as in [1, sec. 5.1] by

$$
\mathbb{P}_{f^{-1}(\mathcal{B})}(n \cdot(b \circ f))=n\left(\mathbb{P}_{\mathcal{B}} b\right) \circ f .
$$

Moving to the Eulerian form of the projected system, we find that we have constructed, for small $G$ and $\Omega$ a solution $\left(\phi, U, \psi_{i}, h_{i j}\right)$ of the following set of projected equations, which is conveniently written in terms of the stress energy components $\tau, \tau_{i}$, and $\tau_{i j}$.

$$
\begin{aligned}
& \mathbb{P}_{f^{-1}(\mathcal{B})}\left(D^{j} \tau_{i j}-2 \omega_{i j} \tau^{j}+\left(D_{i} U\right)\left(e^{-4 U} \tau+\tau_{k}^{k}\right)\right)=0, \\
& \left.\tau_{i j} n^{j}\right|_{\partial f^{-1}(\mathcal{B})}=0, \\
& \Delta_{h} U=4 \pi G \chi_{f^{-1}(\mathcal{B})}\left(e^{-4 U} \tau+\tau_{k}{ }^{k}\right)-e^{4 U} \omega_{k l} \omega^{k l}, \\
& D^{i}\left(e^{4 U} \omega_{i j}\right)+\frac{1}{2} D_{j}\left(e^{4 U} D^{i} \psi_{i}\right)=8 \pi G \chi_{f^{-1}(\mathcal{B})} \tau_{j} \text {, } \\
& G_{i j}-D_{(i} V_{j)}+\frac{1}{2} h_{i j} D_{l} V^{l}=8 \pi G\left(\chi_{f^{-1}(\mathcal{B})} \tau_{i j}+\Theta_{i j}+\Omega_{i j}\right) .
\end{aligned}
$$

Let $Y=\left(f^{A}, U, \psi_{i}, h_{i j}\right)$ be the Eulerian form of the solution to the projected form of the material field equations constructed in Section 3.1. From Proposition 3.1, the solution is unique. For the purposes here, we shall need to make the uniqueness property somewhat more explicit. An analysis of the proof of Proposition 3.1 proves the following corollary.

COROLlaRY 4.2 Let the body domain $\mathcal{B}$ with metric $\delta_{\mathcal{B}}$ be given, with the corresponding background metric $\hat{\delta}$ on $M=\mathbb{R}_{\mathcal{S}}^{3}$, and fix a point $X_{0}$ in $\mathcal{B}$ and a vector field $\eta^{A}$ on $\mathcal{B}$. Then the Eulerian form $Y=\left(f^{A}, U, \psi, h\right)$ of the solution to the reduced projected system for a self-gravitating, rotating, elastic body defines a function of the form

$$
Y=Y\left(G, \Omega ;\left[\mathcal{B}, \delta_{\mathcal{B}}, \hat{\delta}, X_{0}, \eta\right]\right)
$$

\subsection{Equivariance}

We now analyze some of the consequences of the constitutive conditions imposed in Section 2.10. Recall that, particularly in view of frame indifference and 
homogeneity and the discussion in Section 2.10, the reduced stored energy function $\rho$ is of the form

$$
\rho=\rho[f, g ; \eta, \Omega],
$$

where $f^{A}$ is the configuration, $g$ is used as shorthand for the fields $U, \psi_{i}$, and $h_{i j}$ on $M$ parametrizing the spacetime metric $g_{\mu \nu}$, and $\eta^{i}$ is the axial vector field on $M$ specified in Section 2.10. Let $\sigma$ be a spatial diffeomorphism, i.e., $t \circ \sigma=t$. Then by frame indifference (i.e., general covariance), we have

$$
\rho\left[f \circ \sigma ; \sigma^{*} g ; \sigma_{*} \eta, \Omega\right]=\rho[f, g ; \eta, \Omega] \circ \sigma .
$$

Further, as a consequence of the isotropy of the elastic body, for any isometry $\Sigma$ of $\left(\mathcal{B}, \delta_{\mathcal{B}}\right)$, we have

$$
\rho[\Sigma \circ f, g ; \eta, \Omega]=\rho[f, g ; \eta, \Omega] .
$$

The transformation properties stated in (4.3) and (4.4) give the following lemma:

Lemma 4.3 Let $\left(f^{A}, U, \psi_{i}, h_{i j}\right)$ be as in Corollary 4.2. Let $\Sigma$ be a diffeomorphism of $\mathcal{B}$ leaving the data $\left(X_{0}, \delta_{A B}, \eta^{A}\right)$ invariant. Then the diffeomorphism $\sigma$ of $M$ defined by requiring that $\mathbf{i} \circ \Sigma=\sigma \circ \mathbf{i}$ on all of $\mathbb{R}_{\mathcal{B}}^{3}$ is an isometry in the sense that it leaves all of $\left(f^{A}, U, \psi_{i}, h_{i j}, \eta^{i}\right)$ invariant.

PROOF: First note that $\sigma$ is by construction an isometry of the flat background metric $\hat{\delta}$ entering the projected, harmonically reduced field equations and that $\left(\sigma_{*} \eta\right)^{i}=\eta^{i}$ trivially from the construction of $\eta^{i}$. Using these facts together with the equivariance property expressed in (4.3) and (4.4), we have that

$$
\left(\left(\Sigma^{-1} \circ f \circ \sigma\right)^{A}, \sigma^{*} U,\left(\sigma^{*} \psi\right)_{i},\left(\sigma^{*} h\right)_{i j}\right)
$$

is a solution with the same data. By the uniqueness property made explicit in Corollary 4.2, we then have

$$
\left(\left(\Sigma^{-1} \circ f \circ \sigma\right)^{A}, \sigma^{*} U,\left(\sigma^{*} \psi\right)_{i},\left(\sigma^{*} h\right)_{i j}\right)=\left(f^{A}, U, \psi_{i}, h_{i j}\right) .
$$

If $\sigma$ is as in Lemma 4.3, then we also have

$$
\sigma^{*} \tau=\tau, \quad\left(\sigma^{*} \tau\right)_{i}=\tau_{i}, \quad\left(\sigma^{*} \tau\right)_{i j}=\tau_{i j} .
$$

Lemma 4.3 has the following corollary, which will play an important role in the proof of orthogonal transitivity (see Section 4.5 below):

COROLlary 4.4 Let $\left(f^{A}, U, \psi_{i}, h_{i j}\right)$ be as in Corollary 4.2. Let $\Sigma$ be an isometry of $\left(\mathcal{B}, \delta_{\mathcal{B}}\right)$ such that $\Sigma\left(X_{0}\right)=X_{0}$ and $\left(\Sigma_{*} \eta\right)^{A}=-\eta^{A}$, and let $\sigma$ be a diffeomorphism of $M$ such that $\mathbf{i} \circ \Sigma=\sigma \circ \mathbf{i}$ on all of $\mathbb{R}_{\mathcal{B}}^{3}$. Then $\sigma$ is an isometry of $h_{i j}$, and we have

$$
\left(\Sigma^{-1} \circ f^{A} \circ \sigma, \sigma^{*} U,\left(\sigma^{*} \psi\right)_{i},\left(\sigma^{*} h\right)_{i j}\right)=\left(f^{A}, U,-\psi_{i}, h_{i j}\right) .
$$


PROOF: The transformation $\psi_{i} \rightarrow-\psi_{i}, \eta^{A} \rightarrow-\eta^{A}$ leaves $H^{A B}$ and hence all the field equations invariant. Therefore it maps a solution to another solution. By uniqueness it follows that the solution with data $\mathcal{B}, \delta_{\mathcal{B}}, \hat{\delta},-\eta^{A}, G$, and $\Omega$ is given by $\left(f^{A}, U,-\psi_{i}, h_{i j}\right)$. The result follows.

Recall that the reference state is axially symmetric; i.e., $\eta^{A}$ is an axial Killing vector in Euclidean space leaving $\mathcal{B}$ invariant. Denoting by $\Sigma$ the flow of $\eta^{A}$ and correspondingly using $\sigma$ to denote the flow of $\eta^{i}$, we have the following infinitesimal version of Lemma 4.3:

Lemma 4.5 Assume that $\mathcal{B}$ is axially symmetric with axial Killing field $\eta^{A}$, as discussed in Section 2.10. Then

$$
f^{A}{ }_{, i}(x) \eta^{i}(x)=\eta^{A}(f(x)),
$$

i.e.,

$$
\eta^{i} \partial_{i}=f^{*}\left(\eta^{A} \partial_{A}\right)
$$

and

$$
\begin{aligned}
\mathcal{L}_{\eta} U & =0, \\
\mathcal{L}_{\eta} \psi_{i} & =0, \\
\mathcal{L}_{\eta} h_{i j} & =0 .
\end{aligned}
$$

By the antisymmetry of $\omega_{i j}$ we have, using (2.27), that

$$
\mathcal{L}_{\eta}\left(e^{-2 U} \alpha\right)=0 \text {. }
$$

Furthermore, from (4.7) applied to the flow of $\eta^{i}$, we infer that

$$
\mathcal{L}_{\eta} \tau=0, \quad \mathcal{L}_{\eta} \tau_{i}=0, \quad \mathcal{L}_{\eta} \tau_{i j}=0 .
$$

\subsection{Divergence Identities}

Now turn back to equation (2.33). Taking the divergence of this equation and using (4.11a), (4.11c), (4.12), (4.13), and (4.2a) gives

$$
\begin{aligned}
0= & \left(1-\Omega e^{-2 U} \alpha\right) D^{i} \tau_{i}-\Omega D^{i}\left(e^{-2 U} \alpha\right) \tau_{i}+2 \Omega \eta^{j} \omega_{j i} \tau^{i} \\
& +\Omega \eta^{j}\left(\mathbb{I}_{f^{-1}(\mathcal{B})}-\mathbb{P}_{f^{-1}(\mathcal{B})}\right)\left[D^{i} \tau_{i j}-2 \omega_{j i} \tau^{i}\right. \\
= & \left.+\left(D_{j} U\right)\left(e^{-4 U} \tau+\tau_{k}{ }^{k}\right)\right] \\
& +\Omega \eta^{j}\left(\mathbb{I}_{f^{-1}(\mathcal{B})}-\mathbb{P}_{f^{-1}(\mathcal{B})}\right)\left[D^{i} \tau_{i j}-2 \omega_{j i} \tau^{i}\right. \\
& \left.+\left(D_{j} U\right)\left(e^{-4 U} \tau+\tau_{k}{ }^{k}\right)\right] \chi_{f^{-1}(\mathcal{B})}
\end{aligned}
$$

by using (2.26b) for (4.15).

It also follows directly from (2.33) and the fact that $\eta^{i}$ is parallel to the boundary of $f^{-1}(\mathcal{B})$ that the boundary condition

$$
\left.\tau_{i} n^{i}\right|_{f^{-1}(\partial \mathcal{B})}=0
$$


holds. Let $W=e^{4 U} D^{i} \psi_{i}$. The first term in the left-hand side of (4.2d) is the divergence of a 2-form, and therefore its divergence is 0 . Hence, taking the divergence of both sides of (4.2d) and using the fact that (4.16) holds for the case of an axisymmetric body, gives the identity

$$
\Delta_{h} W=16 \pi G \chi_{f^{-1}(\mathcal{B})} D^{i} \tau_{i} .
$$

Equation (4.15) gives the form of the right-hand side in (4.17). Let

$$
L V_{i}=\Delta_{h} V_{i}+{R_{i}}^{k} V_{k}
$$

and note

$$
D^{j}\left(D_{(i} V_{j)}-\frac{1}{2} h_{i j} D_{k} V^{k}\right)=\frac{1}{2} L V_{i} .
$$

Using (2.18) and (2.19) we find after taking the divergence of both sides of (4.2e), when $G \neq 0$, that

$$
L V_{i}=-16 \pi G \chi_{f^{-1}(\mathcal{B})}\left[D^{j} \tau_{i j}+\left(D_{i} U\right)\left(e^{-4 U} \tau+\tau_{k}^{k}\right)\right]+4 \omega_{i k} D^{j}\left(e^{4 U} \omega_{j}^{k}\right)
$$

and by using (4.2d) and (4.2a)

$$
\begin{aligned}
&=-16 \pi G\left[D^{j} \tau_{i j}+\left(D_{i} U\right)\left(e^{-4 U} \tau+\tau_{k}{ }^{k}\right)\right] \chi_{f^{-1}(\mathcal{B})} \\
&+4 \omega_{i j}\left[8 \pi G \chi_{f^{-1}(\mathcal{B})} \tau^{j}-\frac{1}{2} D^{j} W\right] \\
&+16 \pi G \mathbb{P}_{f^{-1}(\mathcal{B})}\left[D^{j} \tau_{i j}+\left(D_{i} U\right)\left(e^{-4 U} \tau+\tau_{k}{ }^{k}\right)-2 \omega_{i j} \tau^{j}\right] \chi_{f^{-1}(\mathcal{B})} \\
&=-16 \pi G\left(\mathbb{I}_{f-1(\mathcal{B})}-\mathbb{P}_{f^{-1}(\mathcal{B})}\right)\left[D^{j} \tau_{i j}+\left(D_{i} U\right)\left(e^{-4 U} \tau+\tau_{k}^{k}\right)\right. \\
&\left.-2 \omega_{i j} \tau^{j}\right] \chi_{f^{-1}(\mathcal{B})}-2 \omega_{i j} D^{j} W .
\end{aligned}
$$

Let

$$
\mathcal{Z}_{i}=-16 \pi G\left[D^{j} \tau_{i j}+\left(D_{i} U\right)\left(e^{-4 U} \tau+\tau_{k}^{k}\right)-2 \omega_{i j} \tau^{j}\right] .
$$

Then we have the following system of equations for $W$ and $V_{i}$ :

$$
\begin{gathered}
\left(1-\Omega e^{-2 U} \alpha\right) \Delta W=\Omega \eta^{j}\left(\mathbb{I}_{f^{-1}(\mathcal{B})}-\mathbb{P}_{f^{-1}(\mathcal{B})}\right) \mathcal{Z}_{j} \chi_{f^{-1}(\mathcal{B})}, \\
L V_{i}=\left(\mathbb{I}_{f^{-1}(\mathcal{B})}-\mathbb{P}_{f^{-1}(\mathcal{B})}\right) \mathcal{Z}_{i} \chi_{f^{-1}(\mathcal{B})}-2 \omega_{i j} D^{j} W .
\end{gathered}
$$

Arguing as in the proof of [1, lemma 5.7], we have that

$$
\left(\mathbb{I}_{f^{-1}(\mathcal{B})}-\mathbb{P}_{f^{-1}(\mathcal{B})}\right) \mathcal{Z}_{i} \chi_{f^{-1}(\mathcal{B})}=n\left(\zeta_{i} \circ f\right) \chi_{f^{-1}(\mathcal{B})}
$$

for some $\zeta_{i}$ that is a Killing field in $\mathcal{B}$. Hence we have the equations

$$
\begin{gathered}
\left(1-\Omega e^{-2 U} \alpha\right) \Delta W=\Omega \eta^{j} n\left(\zeta_{j} \circ f\right) \chi_{f^{-1}(\mathcal{B})}, \\
L V_{i}=n\left(\zeta_{i} \circ f\right) \chi_{f^{-1}(\mathcal{B})}-2 \omega_{i j} D^{j} W .
\end{gathered}
$$




\subsection{Main Theorem}

We are now able to prove the following:

THEOREM 4.6 For sufficiently small values of $G$ and $\Omega$, with $G$ nonzero, the solution to the reduced, projected system of equations for a stationary, rotating, elastic, self-gravitating body (3.4) is a solution to the full system of equations (2.17) for a stationary, rotating, elastic, self-gravitating body, together with the integrability conditions of Section 2.5. In particular, this solution corresponds to a pair $\left(f^{A}, g_{\mu \nu}\right)$ that solves the full Einstein equations $G_{\mu \nu}=8 \pi G T_{\mu \nu}$.

Remark 4.7. The solutions constructed in Theorem 4.6 are static exactly when $\Omega=0$.

PROOF: Using the estimate of Corollary 4.1 and the multiplication properties of the weighted Sobolev spaces (cf. [1, sec. 2.3]), one checks that

$$
\omega_{i j} \in W_{\delta-1}^{1, p}, \quad \Omega_{i j} \in W_{2 \delta-2}^{1, p}, \quad \Theta_{i j} \in W_{2 \delta-2}^{1, p},
$$

with corresponding estimates. We now use (4.2e) for $h_{i j}$ in the equivalent form (2.42), equation (4.2d) for $\psi_{i}$ (making use of equation (2.40) to express it in a form suitable for estimates), as well as (4.2c) for $U$. The result is that the conclusion of [1, lemma 5.2] for $h_{i j}$ also holds in the present case, namely,

$$
h_{i j}=\delta_{i j}+\frac{\gamma_{i j}}{r}+h_{(2) i j}
$$

for constants $\gamma_{i j}$, with $h_{(2) i j} \in W_{2 \delta}^{2, p}$. For sufficiently small $G$ and $\Omega$, we have the estimate

$$
\left\|h_{(2) i j}\right\|_{W_{2 \delta}^{2, p}}+\|\gamma\| \leq C\left(\left\|h_{i j}-\delta_{i j}\right\|_{W_{\delta}^{2, p}}+\|\phi-\mathbf{i}\|_{W^{2, p}(\mathcal{B})}\right) .
$$

For brevity, we shall in what follows write estimates of the above form using $\left\|Z-Z_{0}\right\|_{B_{1}}$ where the norm refers to that induced from the Banach spaces used in defining the space $B_{1}$; cf. Section 3 . We shall further write inequalities of the form $a \leq C b$ where $C$ is a constant that is uniformly bounded for small $G$ and $\Omega$ as $a \lesssim b$.

Given this result about the asymptotics of $h_{i j}$, the conclusion of [1, lemma 5.4] concerning $V_{i}$ holds, and hence also the partial integration result [1, lemma 5.5] and the estimate of $\left[1\right.$, lemma 5.6]. Now define the operator $\mathbb{Q}: L_{\delta-3}^{p}\left(\mathbb{R}_{\mathcal{S}}^{3}\right) \rightarrow \mathbb{R}^{6}$ as in [1, sec. 5.2]. Given a basis $\left\{\xi_{(\kappa)}\right\}_{\kappa=1}^{6}$ for the space of Killing fields, we set

$$
\mathbb{Q}_{\kappa}\left(z_{i}\right)=\int_{\mathbb{R}_{\mathcal{S}}^{3}}\left(\xi_{(\kappa)}^{i} \circ f\right) z_{i} d \mu_{h}, \quad \kappa=1, \ldots, 6 .
$$

Since $W=e^{4 U} D^{i} \psi_{i}$, the term $\omega_{i j} D^{j} W$ in (4.19b) satisfies $\omega_{i j} D^{j} W \in L_{2 \delta-3}^{p}$ and we have the estimate

$$
\left\|\omega_{i j} D^{j} W\right\|_{L_{\delta-3}^{p}\left(\mathbb{R}_{\mathcal{S}}^{3}\right)} \lesssim\left\|Z-Z_{0}\right\|_{B_{1}}\|n(\zeta \circ f)\|_{L^{p}\left(f^{-1}(\mathcal{B})\right)} .
$$


Recall that from the construction of $\mathbb{Q}$ we have for small $G$ and $\Omega$ the equivalence of norms

$$
\left\|\mathbb{Q} n(\zeta \circ f) \chi_{f^{-1}(\mathcal{B})}\right\|_{\mathbb{R}^{6}} \lesssim\|\zeta\|_{\mathbb{R}^{6}} \lesssim\left\|\mathbb{Q} n(\zeta \circ f) \chi_{f^{-1}(\mathcal{B})}\right\|_{\mathbb{R}^{6}}
$$

where if $\zeta^{i}=\alpha^{i}+\beta^{i}{ }_{j} x^{j},\|\zeta\|_{\mathbb{R}^{6}}$ is defined by

$$
\|\zeta\|_{\mathbb{R}^{6}}^{2}=\sum_{i}\left(\alpha^{i}\right)^{2}+\sum_{i<j}\left(\beta_{j}^{i}\right)^{2}
$$

The analogue of (4.21) also holds for $\|n(\zeta \circ f)\|_{L^{p}\left(f^{-1}(\mathcal{B})\right)}$ due to the properties of $\mathbb{Q}$. Applying $\mathbb{Q}$ to both sides of (4.19b), we have using (4.20) and (4.21),

$$
\begin{aligned}
\|\zeta\|_{\mathbb{R}^{6}} & \lesssim\|\mathbb{Q} L V\|_{\mathbb{R}^{6}}+\|\mathbb{Q} \omega D W\|_{\mathbb{R}^{6}} \\
& \lesssim\|\mathbb{Q} L V\|_{\mathbb{R}^{6}}+\left\|Z-Z_{0}\right\|_{B_{1}}\|\zeta\|_{\mathbb{R}^{6}}
\end{aligned}
$$

and hence

$$
\|\zeta\|_{\mathbb{R}^{6}} \lesssim\|\mathbb{Q} L V\|_{\mathbb{R}^{6}}
$$

Recall that for $G$ and $\Omega$ sufficiently small, we also have due to Corollary 4.1 that $\left\|Z-Z_{0}\right\|_{B_{1}}$ is small. We now have the chain of inequalities for $G$ and $\Omega$ sufficiently small,

$$
\begin{aligned}
\|V\|_{W_{\delta-1}^{2, p}} & \lesssim\|L V\|_{W_{\delta-3}^{2, p}} \\
& \lesssim\|n(\zeta \circ f)\|_{L^{p}\left(f^{-1}(\mathcal{B})\right)}+\|\omega D W\|_{L_{\delta-3}^{p}} \\
& \lesssim\|\zeta\|_{\mathbb{R}^{6}}+\left\|Z-Z_{0}\right\|_{B_{1}}\|\zeta\|_{\mathbb{R}^{6}} \\
& \lesssim\|\zeta\|_{\mathbb{R}^{6}} \\
& \lesssim\|\mathbb{Q} L V\|_{\mathbb{R}^{6}} \quad \text { from (4.22). }
\end{aligned}
$$

By the inequality proved in [1, prop. 5.8] we have

$$
\|\mathbb{Q} L V\|_{\mathbb{R}^{6}} \lesssim\left\|Z-Z_{0}\right\|_{B_{1}}\|V\|_{W_{\delta-1}^{2, p}},
$$

which together with the above gives

$$
\|V\|_{W_{\delta-1}^{2, p}} \lesssim\left\|Z-Z_{0}\right\|_{B_{1}}\|V\|_{W_{\delta-1}^{2, p}} .
$$

By choosing $G$ and $\Omega$ sufficiently small, we can make $\left\|Z-Z_{0}\right\|_{B_{1}}$ small enough so that (4.23) gives the inequality

$$
\|V\|_{W_{\delta-1}^{2, p}} \leq \frac{1}{2}\|V\|_{W_{\delta-1}^{2, p}}
$$

which implies

$$
V=0 \text {. }
$$

Due to the vanishing of $V$, it follows from (4.22) that also $\zeta=0$, and hence we have

$$
W=0 \text {. }
$$


This means that the solution of the projected system of equations (4.2) is actually a solution to the full system of field equations (2.17) for the rotating elastic body, together with the integrability conditions discussed in Section 2.5.

It remains to demonstrate that the solution $\left(f^{A}, U, \psi_{i}, h_{i j}\right)$ to (2.17) constructed in this proof corresponds to a Lorentzian spacetime $\left(\mathcal{M}, g_{\mu \nu}\right)$ solving the Einstein equations for the elastic body. The solution we have found yields via (2.4) a Lorentz metric $g_{\mu \nu}$ at some time $t_{0}$ together with its vanishing first and second time derivatives at $t_{0}$, as well as a configuration $f^{A}$ together with its nonvanishing first time derivative at $t_{0}$. These solve the Einstein equations at $t_{0}$. We extend the spacetime metric off $t_{0}$ by requiring it to be $t$-independent and $f^{A}$ by requiring it to satisfy (2.29) for all times. This constructs a spacetime $\left(\mathcal{M}, g_{\mu \nu}\right)$ that is axisymmetric and stationary and a configuration that is axially symmetric and helical. Thus, by the discussion in Section 2.2, the associated energy momentum tensor is time independent. This shows that $\left(\mathcal{M}, g_{\mu \nu}\right)$ together with the configuration $f^{A}$ provides a solution to the full Einstein equations.

In Theorem 4.6 we have treated the field equations for a rotating, self-gravitating body from a mathematical point of view; in particular, we have not made explict the physical units involved. Suppose we have solutions to the problem for $0<G<$ $G_{0}, 0<\Omega<\Omega_{0}$. Since the equations have a physical origin, there are certain similarity transformations that map solutions into solutions. These transformations arise from the possibility of choosing arbitrary units for time, space, and mass in the physical theory.

The Newton constant $G$ has dimensions of

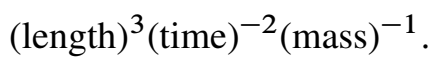

Therefore by an appropriate choice of units, we can bring the numerical value of the physical gravitational constant into the range $0<G<G_{0}$ and interpret our solutions as physical solutions in these particular units.

In our treatment the stored energy function is taken to be independent of $G$ and $\Omega$. Because it contains quantities with physical dimensions, the stored energy in our family of solutions will change under the similarity transformation. Nevertheless, we have shown the existence of solutions that can be interpreted physically.

We remark that our theorem does not cover the case of large, self-gravitating systems like neutron stars, because for these there is no "nearby" relaxed configuration without gravity.

\subsection{Orthogonal Transitivity}

Let $\left(\mathcal{M}, g_{\mu \nu}\right)$ be a stationary spacetime containing a rotating elastic body as constructed in Theorem 4.6. We have shown in Section 4 that $\left(\mathcal{M}, g_{\mu \nu}\right)$ admits a two-parameter, abelian group of isometries, generated by the Killing fields $\xi^{\mu}$ and $\eta^{\mu}$. In fact, since $\eta^{\mu}$ is the pullback of the axial vector field acting on the body, the group can be taken to be the cylinder $\mathbb{R} \times \mathbb{S}^{1}$. The question arises if this group acts orthogonally transitively on $\mathcal{M}$, as is the case for perfect fluids. Recall that 
a group acts orthogonally transitively if the the distribution perpendicular to the generators of the group action is Frobenius integrable.

Define $\omega_{\mu \nu \lambda}=3 \xi_{[\mu} \nabla_{\nu} \xi_{\lambda]}$ and let $\omega^{\prime}{ }_{\mu \nu \lambda}$ be defined with respect to $\eta_{\mu}$ in an analogous manner. Orthogonal transitivity is equivalent to the conditions

$$
\begin{aligned}
& \eta_{[\rho} \omega_{\mu \nu \lambda]}=0, \\
& \xi_{[\rho} \omega^{\prime}{ }_{\mu \nu \lambda]}=0 ;
\end{aligned}
$$

see $[3,(2.53)]$. The spacetimes constructed in this paper have metrics that fail to be smooth at the boundary of the body $f^{-1}(\mathcal{B})$.

PROPOSITION 4.8 Let $\left(\mathcal{M}, g_{\mu \nu}\right)$ be a stationary spacetime containing a rotating elastic body as in Theorem 4.6 with stationary and axial Killing fields $\xi^{\mu}$ and $\eta^{\mu}$, respectively. Then if $\Omega>0$ is sufficiently small, equation (4.24) holds in $\mathcal{M}$.

PROOF: The conditions (4.24) can be restated in the space manifold $M$ as

$$
\begin{aligned}
e^{2 U} \eta_{[i} \omega_{j k]} & =0, \\
e^{-2 U} \eta_{[i} D_{j} \eta_{k]}+\alpha \eta_{[i} \omega_{j k]} & =0 .
\end{aligned}
$$

Here $\omega_{i j}=\partial_{[i} \psi_{j]}$ and $\alpha=\xi^{\mu} \eta_{\mu}$, as above. Equations [3, (2.60),(2.61)] are equivalent to (4.25) but written in terms of a different representation of the spacetime metric.

By [3, equations (2.51)-(2.52)], we have

$$
\begin{aligned}
4 \nabla^{\mu}\left(\eta_{[\rho} \omega_{\mu \nu \lambda]}\right) & =6 \xi^{\mu} R_{\mu[\nu} \xi_{\lambda} \eta_{\rho]}, \\
4 \nabla^{\mu}\left(\xi_{[\rho} \omega^{\prime}{ }_{\mu \nu \lambda]}\right) & =-6 \eta^{\mu} R_{\mu[\nu} \xi_{\lambda} \eta_{\rho]} .
\end{aligned}
$$

It follows that

$$
\nabla^{\mu}\left(\eta_{[\rho} \omega_{\mu \nu \lambda]}-\Omega \xi_{[\rho} \omega^{\prime}{ }_{\mu \nu \lambda]}\right)=12 \pi G\left(\xi^{\mu}+\Omega \eta^{\mu}\right) T_{\mu[\nu} \xi_{\lambda} \eta_{\rho]} .
$$

Since the velocity vector $u^{\mu}=b^{-1}\left(\xi^{\mu}+\Omega \eta^{\mu}\right)$ (see equation (2.28)) is an eigenvector of the stress energy tensor (cf. equation (2.1)), the right-hand side of (4.27) is 0 . The left-hand side of (4.27) is the divergence of a 4-form, i.e., in terms of the exterior derivative and the Hodge dual, we have an equation of the form $\star \mathrm{d} \star \alpha=0$. In particular, $\star \alpha$ is a scalar function that is constant, $\mathrm{d} \star \alpha=0$. In the situation under consideration, $\alpha$ vanishes on the axis $x^{1}=x^{2}=0$, and hence it is 0 everywhere. In this argument we made use of the fact that $u^{\mu}$ is well-defined in all of $f^{-1}(\mathcal{B})$. This holds for sufficiently small values of $\Omega$, since then the vector field $\xi^{\mu}+\Omega \eta^{\mu}$ is timelike in all of $f^{-1}(\mathcal{B})$. In terms of the space manifold $M$, we have shown that

$$
\Omega \eta_{[i} D_{j} \eta_{k]}-e^{4 U}\left(1-\Omega e^{-2 U} \alpha\right) \eta_{[i} \omega_{j k]}=0 .
$$

This can of course also be checked directly from the three-dimensional field equations. Note that relation (4.28) becomes vacuous in the static case $\Omega=0$.

By the above argument we have shown that the two equations (4.25) are linearly dependent if $\Omega \neq 0$. Thus, in order to show that both equations in (4.25) hold, it is 
sufficient to show that $\eta_{[i} D_{j} \eta_{k]}=0$. To see this, we argue as follows: It follows from the axisymmetry of the body that there is a discrete isometry $\Sigma$ of $\left(\mathcal{B}, \delta_{\mathcal{B}}\right)$, consisting of reflections in planes containing the $X^{3}$-axis, that maps $\eta^{A}$ to $-\eta^{A}$. An explicit choice of $\Sigma$ is given by

$$
\Sigma\left(X^{1}, X^{2}, X^{3}\right)=\left(-X^{1}, X^{2}, X^{3}\right) .
$$

By Corollary 4.4 and the construction of $\eta^{i}$, we have that the diffeomorphism $\sigma$ of $M$ defined by $\Sigma \circ \mathbf{i}=\sigma \circ \mathbf{i}$ is an isometry of $h_{i j}$, which has the property that $\left(\sigma_{*} \eta\right)^{i}=-\eta^{i}$. We can now conclude that reflections at planes through the $x^{3}$-axis preserve both $U$ and $h_{i j}$ and send both $\psi_{i}$ and $\eta^{i}$ to their respective negatives. So, in particular, these transformations preserve vectors tangent to these planes, and since they send $\eta^{i}$ to $-\eta^{i}$ and preserve inner products, $\eta^{i}$ has to be orthogonal to these planes. Consequently, $\eta^{i}$ is hypersurface orthogonal, i.e.,

$$
\eta_{[i} D_{j} \eta_{k]}=0 .
$$

It follows, using (4.28), that (4.25) holds.

Remark 4.9. Recall the identity valid for Killing vectors

$$
3 D^{i}\left(\eta_{[i} D_{j} \eta_{k]}\right)=2 \eta_{[j} R_{k] l} \eta^{l} .
$$

Inserting (4.30) into (2.14c), using (2.27), (2.26a), and finally (4.28), we obtain

$$
3 D^{i}\left[\left(1-\Omega e^{-2 U} \alpha\right)^{1 / 3} \eta_{[i} D_{j} \eta_{k]}\right]=16 \pi G\left(1-\Omega e^{-2 U} \alpha\right)^{1 / 3} \eta^{i} \tau_{i}\left[j \eta_{k]} .\right.
$$

Thus we have inferred that $\eta^{i}$ is an eigenvector of the stress tensor. This latter fact could have also been shown directly from the reflection symmetry without using the Einstein equations.

Remark 4.10. In the case of a smooth spacetime, it follows from (4.24) and the Frobenius theorem that the distribution perpendicular to $\xi^{\mu}$ and $\eta^{\mu}$ is integrable in the sense that there are smooth 2-surfaces in $\mathcal{M}$ orthogonal to the span of $\xi^{\mu}$ and $\eta^{\mu}$. The spacetimes constructed in Theorem 4.6 have been shown to be $W_{\text {loc }}^{2, p}$. Although the spacetimes containing a rotating body can in fact be shown to be real analytic away from the boundary of the body, $f^{-1}(\partial \mathcal{B})$, a further analysis is needed to show that an appropriate version of the Frobenius theorem applies. This question will be studied in a later paper.

\section{Appendix: Proof of Lemma 2.1}

We have $\Lambda=e^{3 U} \rho$. From

$$
T_{\mu \nu}=2 \frac{\partial \Lambda}{\partial g^{\mu \nu}}-\Lambda g_{\mu \nu},
$$

we get

$$
\frac{\partial \Lambda}{\partial g^{\mu \nu}}=\frac{1}{2}\left(T_{\mu \nu}+\Lambda g_{\mu \nu}\right) .
$$


Using a form of $g^{\mu \nu}$ (cf. (2.5)), we have

$$
\begin{aligned}
& \frac{\partial g^{\mu \nu}}{\partial h^{i j}} \partial_{\mu} \partial_{\nu}=e^{2 U}\left(\partial_{i} \partial_{j}-\psi_{i} \psi_{j} \partial_{t}^{2}\right), \\
& \frac{\partial g^{\mu \nu}}{\partial \psi^{i}} \partial_{\mu} \partial_{\nu}=e^{2 U}\left(-2 \partial_{i} \partial_{t}+2 \psi_{i} \partial_{t}^{2}\right), \\
& \frac{\partial g^{\mu \nu}}{\partial U} \partial_{\mu} \partial_{\nu}=2 g^{\mu \nu} \partial_{\mu} \partial_{\nu}+4 e^{-2 U} \partial_{t}^{2} .
\end{aligned}
$$

Define $\tau, \tau_{i}$, and $\tau_{i j}$ by

$$
T_{\mu \nu}=\tau\left(d t+\psi_{i} d x^{i}\right)^{2}+2 \tau_{j} d x^{j}\left(d t+\psi_{i} d x^{i}\right)+\tau_{i j} d x^{i} d x^{j} .
$$

Then

$$
\begin{aligned}
T_{i j} & \left.=\tau_{i j}+2 \tau_{(i} \psi_{j}\right)+\tau \psi_{i} \psi_{j}, \\
T_{0 i} & =\tau_{i}+\tau \psi_{i}, \\
T_{00} & =\tau, \\
T_{\mu}{ }^{\mu} & =-e^{-2 U} \tau+e^{2 U} \tau_{\ell}^{\ell} .
\end{aligned}
$$

We calculate

$$
\begin{aligned}
e^{U}\left(2 \frac{\partial \rho}{\partial h^{i j}}-\rho h_{i j}\right) & =e^{-2 U}\left(2 \frac{\partial \Lambda}{\partial h^{i j}}-\Lambda h_{i j}\right) \\
& =e^{-2 U}\left(2 \frac{\partial \Lambda}{\partial g^{\mu \nu}} \frac{\partial g^{\mu \nu}}{\partial h^{i j}}-\Lambda h_{i j}\right) \\
& =e^{-2 U}\left[\left(T_{\mu \nu}+\Lambda g_{\mu \nu}\right) \frac{\partial g^{\mu \nu}}{\partial h^{i j}}-\Lambda h_{i j}\right] \\
& =T_{i j}-T_{00} \psi_{i} \psi_{j}+\Lambda\left(g_{i j}-g_{00} \psi_{i} \psi_{j}\right)-\Lambda e^{-2 U} h_{i j} \\
& =\tau_{i j}, \\
e^{U} \frac{\partial \rho}{\partial \psi^{i}} & =e^{-2 U} \frac{\partial \Lambda}{\partial \psi^{i}} \\
& =e^{-2 U} \frac{\partial \Lambda}{\partial g^{\mu \nu}} \frac{\partial g^{\mu \nu}}{\partial \psi^{i}} \\
& =e^{-2 U} \frac{1}{2}\left(T_{\mu \nu}+\Lambda g_{\mu \nu}\right) \frac{\partial g^{\mu \nu}}{\partial \psi^{i}} \\
& =-T_{i 0}+\psi_{i} T_{00}-\Lambda g_{i 0}+\Lambda \psi_{i} g_{00} \\
& =-\tau_{i},
\end{aligned}
$$




$$
\begin{aligned}
e^{U}\left(\frac{\partial \rho}{\partial U}+\rho\right) & =e^{-2 U}\left(\frac{\partial \Lambda}{\partial g^{\mu \nu}} \frac{\partial g^{\mu \nu}}{\partial U}-2 \Lambda\right) \\
& =e^{-2 U}\left(\frac{1}{2}\left(T_{\mu \nu}+\Lambda g_{\mu \nu}\right) \frac{\partial g^{\mu \nu}}{\partial U}-2 \Lambda\right) \\
& =e^{-2 U}\left(\left(T_{\mu \nu}+\Lambda g_{\mu \nu}\right)\left(g^{\mu \nu}+2 e^{-2 U} \delta^{\mu}{ }_{0} \delta^{\nu}{ }_{0}\right)-2 \Lambda\right) \\
& =e^{-2 U}\left(T_{\mu}^{\mu}+4 \Lambda+2 e^{-2 U} T_{00}+2 e^{-2 U} \Lambda g_{00}-2 \Lambda\right) \\
& =e^{-2 U}\left(T_{\mu}{ }^{\mu}+2 e^{-2 U} T_{00}\right) \\
& =e^{-4 U} \tau+\tau_{\ell}{ } .
\end{aligned}
$$

Acknowledgment. LA and RB thank the Mittag-Leffler Institute, Djursholm, Sweden, where part of this paper was written, for hospitality and support. LA was supported in part by the National Science Foundation under Grants DMS 0407732 and DMS 0707306. RB was supported in part by Fonds zur Förderung der Wissenschaftlichen Forschung, project no. P20414-N16.

\section{Bibliography}

[1] Andersson, L.; Beig, R.; Schmidt, B. G. Static self-gravitating elastic bodies in Einstein gravity. Comm. Pure Appl. Math. 61 (2008), no. 7, 988-1023.

[2] Beig, R.; Heinzle, J. M.; Schmidt, B. G. Helically symmetric $N$-particle solutions in scalar gravity. Phys. Rev. Lett. 98 (2007), no. 12, 121102.

[3] Beig, R.; Schmidt, B. G. Time-independent gravitational fields. Einstein's field equations and their physical implications, 325-372. Lecture Notes in Physics, 540. Springer, Berlin, 2000.

[4] Beig, R.; Schmidt, B. G. Relativistic elasticity. Classical Quantum Gravity 20 (2003), no. 5, 889-904.

[5] Beig, R.; Schmidt, B. G. Relativistic elastostatics. I. Bodies in rigid rotation. Classical Quantum Gravity 22 (2005), no. 11, 2249-2268.

[6] Carter, B.; Samuelsson, L. Relativistic mechanics of neutron superfluid in (magneto)elastic star crust. Classical Quantum Gravity 23 (2006), no. 17, 5367-5388.

[7] Frauendiener, J.; Kabobel, A. The static spherically symmetric body in relativistic elasticity. Classical Quantum Gravity 24 (2007), no. 18, 4817-4837.

[8] Heilig, U. On Lichtenstein's analysis of rotating Newtonian stars. Ann. Inst. H. Poincaré Phys. Théor. 60 (1994), no. 4, 457-487.

[9] Heilig, U. On the existence of rotating stars in general relativity. Comm. Math. Phys. 166 (1995), no. 3, 457-493.

[10] Karlovini, M.; Samuelsson, L. Elastic stars in general relativity. I. Foundations and equilibrium models. Classical Quantum Gravity 20 (2003), no. 16, 3613-3648.

[11] Karlovini, M.; Samuelsson, L. Elastic stars in general relativity. IV. Axial perturbations. Classical Quantum Gravity 24 (2007), no. 13, 3171-3189.

[12] Kijowski, J.; Magli, G. Unconstrained variational principle and canonical structure for relativistic elasticity. Rep. Math. Phys. 39 (1997), no. 1, 99-112.

[13] Kundt, W.; Trümper, M. Orthogonal decomposition of axi-symmetric stationary spacetimes. Z. Physik 192 (1966), 419-422. 
[14] Lindblom, L. Stationary stars are axisymmetric. Astrophys. J. 208 (1976), no. 3, part 1, 873880.

[15] Lindblom, L. On the symmetries of equilibrium stellar models. Philos. Trans. Roy. Soc. London Ser. A 340 (1992), no. 1658, 353-364.

[16] Marsden, J. E.; Hughes, T. J. R. Mathematical foundations of elasticity. Dover, New York, 1994.

[17] Papapetrou, A. Champs gravitationnels stationnaires à symétrie axiale. Ann. Inst. H. Poincaré (A) Phys. Théor. 4 (1966), no. 2, 83-105.

[18] Stephani, H.; Kramer, D.; MacCallum, M.; Hoenselaers, C.; Herlt, E. Exact solutions of Einstein's field equations. 2nd ed. Cambridge Monographs on Mathematical Physics. Cambridge University Press, Cambridge, 2003.

[19] Tahvildar-Zadeh, A. S. Relativistic and nonrelativistic elastodynamics with small shear strains. Ann. Inst. H. Poincaré Phys. Théor. 69 (1998), no. 3, 275-307.

LARS ANDERSSON

Max-Planck-Institut

für Gravitationsphysik

Albert Einstein Institut

Am Mühlenberg 1

D-14476 Golm

GERMANY

E-mail: larsa@math.miami.edu

\section{ROBERT BEIG}

Universität Wien

Gravitational Physics

Faculty of Physics

Boltzmanngasse 5

A-1090 Vienna

AUSTRIA

E-mail: beig@ap.univie.ac.at

\section{BERND G. SCHMIDT}

Max-Planck-Institut für Gravitationsphysik

Albert-Einstein-Institut

Am Mühlenberg 1

D-14476 Golm

GERMANY

E-mail: berndeaei.mpg •de

Received February 2009. 